\title{
Some Remarks on Units in Grothendieck-Witt Rings
}

\author{
Tom Bachmann* \\ LMU Munich, Munich, Germany \\ tom.bachmann@zoho.com
}

December 6, 2017

\begin{abstract}
We establish new structures on Grothendieck-Witt rings, including a $G W(k)$-module structure on the unit group $G W(k)^{\times}$and a presentation of $G W^{\times}$as an infinite $\mathbb{G}_{m}$-loop sheaf. Even though our constructions are motivated by speculations in stable $\mathbb{A}^{1}$-homotopy theory, our arguments are purely algebraic.
\end{abstract}

\section{Contents}

1 Introduction

2 Multiplicative transfers on $G W$

$3 G W$ as a Tambara functor

4 The $G W$-module structure on $G W^{\times}$

5 The sheaf $\underline{G W^{\times}}$and the homotopy module $F_{*}$

6 The logarithm isomorphism

7 Delooping $\underline{G W^{\times}}$

A Recollections on homotopy modules

B Recollections on continuity

\section{Introduction}

The main objects of investigation of this article are the ring-valued functors $X \mapsto G W(X)$ and $X \mapsto$ $\underline{G W}(X)$ and their subfunctors of units $G W^{\times}(X)$ and $\underline{G W^{\times}}(X)$. Recall that for a scheme $X, G W(X)$ is the Grothendieck-Witt ring of $X$ [11], and for $X$ smooth over a perfect field, $\underline{G W}(X)$ is the unramified Grothendieck-Witt ring of $X$ [20, Chapter 3]. The connection is that for $X$ (essentially) smooth local, we have $G W(X)=\underline{G W}(X)$, cf. [21, Theorem A].

Our principal contribution is the following. We show that if $k$ is a field of characteristic not 2, then the group of units $G W^{\times}(k)$ has a canonical structure of a module over $G W(k)$, related to Rost's multiplicative transfer on $G W(k)$. We use this to give a novel presentation of $G W^{\times}(k)$, see Proposition 25. and to construct a homotopy module $T_{*}$ such that $T_{0} \cong \underline{G W^{\times}}$. See Appendix $\AA$ for some recollections regarding homotopy modules.

*present address: Fakultät Mathematik, Universität Duisburg-Essen, Thea-Leymann-Straße 9, 45127 Essen, Germany 
Organisation. We now provide an overview of the article. The remaining subsections of the introduction provide a more leisurely account of some of the key ideas mentioned here.

In Section 2, we recall the results of Rost and his students on multiplicative transfers for the Grothendieck-Witt ring $G W(X)$ [22, 12. Specifically, the multiplicative transfer of Rost is defined using a certain norm functor for modules, also defined by Rost. We show that Rost's norm construction coincides with a more general construction of Ferrand [7, in the situation where both apply.

In Section 3, using this comparison of norm constructions, we show that the assignment $F$ ét/S $\ni$ $X \mapsto G W(X)$ defines a Tambara functor. Here $F e ́ t / S$ denotes the category of finite étale schemes over $S$, and by a Tambara functor on this category we mean the evident extension of the notion from [27]; see Definition 8 for details. Using a result of Tambara [27, Theorem 6.1], this also yields an alternative proof that the norm maps extend from $\operatorname{Iso}(\operatorname{Bil}(\bullet))$ to $G W(\bullet)$.

Section 4 contains our main observation. We show that if $k$ is a field of characteristic not 2 , then the group of units $G W^{\times}(k) \subset G W(k)$ is a module over $G W(k)$, in a unique way that is compatible with the projection formula. By this we mean that if $A / k$ is finite étale, then for $x \in G W(A)$ and $y \in G W^{\times}(k)$ the following formula holds:

$$
y^{t r_{A / k}(x)}=N_{A / k}\left(\left(\left.y\right|_{A}\right)^{x}\right) .
$$

Note that we write the module structure as "exponentiation". This result is Proposition 22, Uniqueness of the $G W(k)$-module structure follows from the fact that as an abelian group, $G W(k)$ is generated by the traces of finite étale algebras, in fact traces of degree at most 2 extensions suffice. This is explained before Proposition 22, Existence/well-definedness is a consequence of Serre's splitting principle; see Lemma 20. In the remainder of that section we establish many simple but useful properties of this $G W(k)$-module structure.

In Section 5 we pass to associated sheaves. Thus we study the unramified sheaf of units $\underline{G W^{\times}} \subset \underline{G W}$. We define a filtration $F_{\bullet} G W^{\times}$, where $x \in F_{n}$ if and only if $x \equiv 1\left(\bmod \underline{I}^{n}\right)$. Here $\underline{I}$ denotes the unramified sheaf of fundamental ideals. We can determine the subquotients $F_{n} \underline{G W} \underline{W}^{\times} / F_{n+1} \underline{G} W^{\times}$and this allows us to prove in Theorem 30 that $\underline{G W^{\times}}$is strictly homotopy invariant. This fixes a gap in a result of Wendt [28]. With this preliminary out of the way, we can extend the module structure from the previous section to obtain a $\underline{G W}$-module structure on $\underline{G W^{\times}}$. Using it we define morphisms $\beta_{n}^{\dagger}: F_{n} \underline{G W^{\times}} \rightarrow\left(F_{n+1} \underline{G W^{\times}}\right)_{-1}$. In Proposition 33 we prove that $\beta_{n}^{\dagger}$ is an isomorphism for $n \geq 2$. In other words, we have constructed a homotopy module $F_{*}$ with $F_{n}=F_{n} \underline{G W^{\times}}$for $n \geq 2$.

In Section 6 we study the homotopy module $F_{*}$. We show that for $* \geq 2$ its canonical $G W$-module structure and cohomological transfers coincide respectively with the module structure on $F_{n} \underline{G W}{ }^{\times}$constructed in the previous section and Rost's multiplicative transfers. In doing so we define an isomorphism of homotopy modules $\log : F_{*} \rightarrow \underline{I}_{t o r}^{*}$. In particular this map turns Rost's multiplicative transfers into the usual additive ones.

In the final Section 7 we put everything together in Theorem 44, There we construct a homotopy module $T_{*}$ with $T_{0} \cong \underline{G W}{ }^{\times}$such that the $\underline{G W}$-module structure and cohomological transfers on $T_{0}$

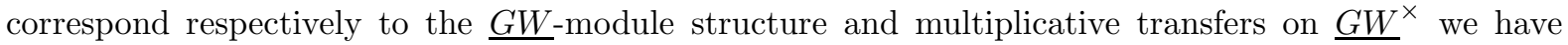
constructed before. Along the way we establish the following exact sequence:

$$
0 \rightarrow \underline{I}^{2} / 2 \underline{I \oplus\langle 2\rangle-1} \longrightarrow \underline{G W} / 2 \oplus \underline{I}_{t o r}^{2} \rightarrow \underline{G W}^{\times} \rightarrow 1 .
$$

Here $i$ is induced from the canonical inclusion $\underline{I}^{2} \rightarrow \underline{G W}$. This appears to be a novel presentation of the group of units of $\underline{G W}$.

The paper concludes with two short appendices. In Appendix $\mathrm{A}$ we recall the basics about homotopy modules. In Appendix B] we recall a well-known continuity result.

Notations and conventions. We make the blanket assumption throughout that $k$ is a field of characteristic different from 2 . When dealing with homotopy modules, we also assume that the base field is perfect, since this is when the theory is most well-behaved.

If $X$ is a scheme and $a \in \mathcal{O}^{\times}(X)$, we denote by $\langle a\rangle \in G W(X)$ the class of the bilinear space with underlying vector bundle $\mathcal{O}_{X}$ and with form $(x, y) \mapsto a x y$.

Acknowledgements. This work would not have been possible without Rost's initiation of the study of multiplicative transfers on $G W[22$ and the detailed computations by his student Wittkop in the case of a quadratic extension [29]. The unpublished preprint of Wendt 28] also was very influential to us, even though we do not end up explicitly re-using any of the results from that paper.

This work has been carried out while the author was a post-doctoral student at LMU Munich. 
The author would like to thank an anonymous referee for an exceptionally thorough review, as a result of which the presentation was improved considerably.

Motivation from $\mathbb{A}^{1}$-homotopy theory. If $E$ is an $E_{\infty}$-ring spectrum (in the classical sense), then $\Omega^{\infty} E$ is an $E_{\infty}$-ring space, and hence the subspace of units $\left(\Omega^{\infty} E\right)^{\times}$is a grouplike $E_{\infty}$-space. Consequently, by classical infinite loop space theory, there is a (unique) connective spectrum $g l_{1}(E)$ such that $\Omega^{\infty} g l_{1}(E) \simeq\left(\Omega^{\infty} E\right)^{\times}$as $E_{\infty}$-spaces.

We are motivated by the question if a similar construction can be possible in stable motivic homotopy theory [17, Section 5]. We thus fix a base field $k$, and we have the adjunction

$$
\Sigma_{+}^{\infty}: \mathbf{H}(k) \leftrightarrows \mathbf{S H}(k): \Omega^{\infty}
$$

where $\mathbf{H}(k)$ denotes the unstable, unpointed motivic homotopy category over $k$, and $\mathbf{S H}(k)$ denotes the $\mathbb{P}^{1}$-stable motivic homotopy category over $k$. One may show that if $E \in \mathbf{S H}(k)$ is an $E_{\infty}$-ring spectrum in the ordinary sense, then it need not be the case that there exists $g l_{1}(E) \in \mathbf{S H}(k)$ such that $\Omega^{\infty}(E)^{\times} \simeq \Omega^{\infty} g l_{1}(E)$, even just as motivic spaces. In other words, the space of units in $E$ need not be an infinite $\mathbb{P}^{1}$-loop space. This does not rule out, however, that there might be a stronger notion of a highly structured ring spectrum in $\mathbf{S H}(k)$ which has this property 1

Being an infinite $\mathbb{P}^{1}$-loop space is a very strong requirement. We are only studying one obstruction in this article: if $X$ is an infinite $\mathbb{P}^{1}$-loop space, then all the homotopy sheaves $\underline{\pi}_{i}(E)$ extend to homotopy modules. Among many other things, this means that they must be modules over $\underline{\pi}_{0}\left(\Omega^{\infty} S^{0}\right)=\underline{G W}$, cf. [17.

Hence the starting point of our investigation: if there is any notion of a highly structured motivic commutative ring spectrum, surely the sphere spectrum must be an example of such an object. As cited above, $\underline{\pi}_{0}(S)=\underline{G W}$ is the sheaf of unramified Grothendieck-Witt groups. Hence if there is to be any hope for a motivic multiplicative infinite loop space theory of this form, the sheaf of units $\underline{G W^{\times}}$must extend to a homotopy module. In particular, for every field $k$, the group of units in the GrothendieckWitt ring of $k$ must be a module over the Grothendieck-Witt ring itself!

The aim of this article is to show that this is indeed the case, and that in fact $\underline{G W} W^{\times}$does extend to a homotopy module. Hence, at least from this perspective, the existence of a motivic multiplicative infinite loop space theory is not ruled out.

The $G W$-module structure. At the first sight, the claim that $G W^{\times}(k)$ should be a module over $G W(k)$ may seem preposterous; at least it did so to the author. Here we try to de-mystify this structure somewhat. First a philosophical remark: one should think of the $G W$-module structure as arising in essentially the same fashion as the $\mathbb{Z}$-module structure on $\mathbb{Z}^{\times}=\{ \pm 1\}$. For this reason, we write the action of $x \in G W(k)$ on $y \in G W^{\times}(k)$ as the "exponentiation" $y^{x}$.

Secondly, here are some formulas. The defining property of this $G W$-module structure is that for $x \in G W^{\times}(k)$ and $A / k$ finite étale, we have $x^{\operatorname{tr}(A)}=N_{A / k}\left(\left.x\right|_{A}\right)$. Here $\operatorname{tr}_{A / k}: G W(A) \rightarrow G W(k)$ is the Scharlau transfer, $N_{A / k}: G W(A) \rightarrow G W(k)$ is the Rost Norm [22, and $\operatorname{tr}(A):=\operatorname{tr}_{A / k}(1)$. Suppose that $A=k(\sqrt{a})$. Then one may check that $\operatorname{tr}(A)=\langle 2\rangle(1+\langle a\rangle)$. From this it follows easily that elements of the form $\operatorname{tr}(A)$ for $[A: k] \leq 2$ generate $G W(k)$ as an abelian group, so we only have to understand these exponents. Fortunately this situation has been studied thoroughly by Wittkop [29], and the following formula is an immediate corollary of his work (see Lemma 16):

$$
(x+y)^{\operatorname{tr}(A)}=x^{\operatorname{tr}(A)}+y^{\operatorname{tr}(A)}+\operatorname{tr}(A) x y .
$$

Here $A / k$ is an extension of degree 2. The trivial extension $A=k \times k$ is allowed, in which case $\operatorname{tr}(A)=2 \cdot\langle 2\rangle=2$ (see e.g. Lemma 42) and the formula is familiar. From this $\left(\right.$ and $\left.N_{A / k}(0)=0\right)$ one also obtains

$$
(-1)^{\operatorname{tr}(A)}=\operatorname{tr}(A)-1 ;
$$

see Proposition 23 part (ii). Finally one may check the following formula for all $x \in G W(k), a \in k^{\times}$:

$$
\langle a\rangle^{x}=\langle a\rangle^{\operatorname{dim}(x)} \text {. }
$$

Together these three formulas in principle allow the computation of $x^{y}$ for any $x \in G W^{\times}(k)$ and $y \in G W(k)$. This is illustrated for example in the proof of Proposition 23 .

\footnotetext{
${ }^{1}$ In fact, in [4 the author and M. Hoyois introduce the notion of normed spectra, which are enhancements of naive $E_{\infty}$-algebras in motivic spectra. If $E$ is a normed spectrum then $\pi_{0}(E)$ acquires multiplicative transfers along finite étale morphisms. The sphere spectrum $S^{0}$ has a unique normed structure, and the multiplicative transfers induced on $\underline{\pi}_{0}\left(S^{0}\right)=\underline{G W}$ are Rost's norms [4, Theorem 10.13]. Unfortunately we do not know if $E$ being a normed spectrum implies that $\left(\Omega^{\infty} \overline{E)^{\times}}\right.$is an infinite $\mathbb{P}^{1}$-loop space.
} 
The logarithm isomorphism. A further surprising property is that at least on some part of $G W^{\times}(k)$, the multiplicative structures can be made equivalent to the additive ones. Let us also try to shed some light on that.

The logarithm map furnishes an isomorphism of abelian groups

$$
\log : F_{2} G W^{\times}(k)=\left(1+I_{\text {tor }}^{2}(k), \times\right) \rightarrow\left(I_{\text {tor }}^{2}(k),+\right) .
$$

This map satisfies

$$
\begin{gathered}
\log (x y)=\log (x)+\log (y), \text { for } x, y \in F_{2} G W^{\times}(k), \\
\log \left(x^{z}\right)=z \log (x), \text { for } z \in G W(k)
\end{gathered}
$$

and

$$
\log \left(N_{A / k}(w)\right)=t r_{A / k}(\log (w))
$$

for $A / k$ finite étale and $w \in F_{2} G W^{\times}(A)$. These three properties are what we mean by turning multiplicative structures into additive ones.

The logarithm map is constructed as follows. Given $x \in F_{2} G W^{\times}(k)$, let $t_{1}, \ldots, t_{m}$ be independent variables. Then consider the element

$$
y:=x^{\left(\left\langle t_{1}\right\rangle-1\right) \ldots\left(\left\langle t_{m}\right\rangle-1\right)} \in G W^{\times}\left(k\left(t_{1}, \ldots, t_{m}\right)\right) .
$$

It follows from the theory of homotopy modules that $y$ may be written as $y=1+\log _{(m)}(x)\left(\left\langle t_{1}\right\rangle-\right.$ $1) \ldots\left(\left\langle t_{m}\right\rangle-1\right)$ for a unique element $\log _{(m)}(x) \in I_{\text {tor }}^{2}(k)$; see Lemma 36. Then $\log (x):=\lim _{m \rightarrow \infty} \log _{(m)}(x)$. This limit makes sense because the sequence is eventually constant; see Theorem 40. See also Remark 37 for a comparison to the logarithm function in real analysis.

Remark on characteristic 2. The theory of Grothendieck-Witt rings in characteristic 2 can be rather different from the other characteristics. However, when working with bilinear forms, many of these differences disappear. A natural question is then if our results can be extended to characteristic 2 . The main obstruction to this is the following:

Lemma 1. Let $\operatorname{char}(k)=2$ and $l / k$ be a finite separable extension. Then the bilinear form $\operatorname{tr}_{l / k}(1) \in$ $G W(k)$ is isomorphic to the trivial form $[l: k]$.

Indeed, recall that we try to define a $G W(k)$-module structure on $G W(k)^{\times}$by requiring that $u^{t r_{l / k}(1)}=N_{l / k}\left(\left.u\right|_{l}\right)$; the above Lemma shows that in characteristic 2 this formula does not put any constraints on a hypothetical module structure at all. Thus our method cannot work in characteristic 2 .

Proof. If $[l: k]=2$ this is checked by direct computation. In general, after an odd degree base change, which induces an injection on $G W$, we may assume that $l / k$ is obtained as a sequence of quadratic extensions; the result follows. See Proposition 17 for a more detailed proof using a similar argument.

\section{Multiplicative transfers on $G W$}

\section{$2.1 \quad$ Split $K$-groups}

Given a scheme $X$, we have the categories $\operatorname{Vect}(X)$ and $\operatorname{Bil}(X)$ of vector bundles on $X$ and vector bundles on $X$ provided with a bilinear form, respectively. By "bilinear form" we shall always mean a symmetric, non-degenerate bilinear form. In other words an object of $\operatorname{Bil}(X)$ is a vector bundle $E$ together with a homomorphism $b_{E}: E \otimes E \rightarrow \mathcal{O}_{X}$, such that (1) $b_{E}$ is symmetric, i.e. $b_{E} \circ \tau_{E}=b_{E}$, where $\tau_{E}: E \otimes E \rightarrow E \otimes E$ is the twist isomorphism, and (2) the homomorphism $b_{E}^{\vee}: E \rightarrow E^{\vee}$ induced by adjunction is an isomorphism. Here $E^{\vee}$ denotes the dual bundle.

Write $I \operatorname{so}(V e c t(X))$ for the set of isomorphism classes of vector bundles; this is an abelian semi-group. Let $K(V e c t(X))^{\oplus}$ be its associated Grothendieck group. It is also known as the direct-sum $K$-theory $K_{0}^{\oplus}(X)$ of $X$. In other words $K(V e c t(X))^{\oplus}$ is obtained as the Grothendieck group of the exact category $V$ ect $(X)$, but where only split exact sequences are allowed in the exact structure. If $X$ is affine, this coincides with the usual group $K_{0}(X)$ (where all exact sequences are allowed in the exact structure), but for general $X$ it does not. We can do the same with $\operatorname{Bil}(X)$ : we get the abelian semi-group $I \operatorname{so}(\operatorname{Bil}(X))$, and the associated Grothendieck group $K(\operatorname{Bil}(X))^{\oplus}$ coincides with the usual Grothendieck-Witt group $G W(X)$ for $X$ affine. 
If $f: X \rightarrow Y$ is a morphism of schemes, there is the usual pushforward $f_{*}: Q \operatorname{Coh}(X) \rightarrow Q \operatorname{Coh}(Y)$. If $f$ is finite locally free (see e.g. [26. Tag 02KA] for a definition) and $V \in Q \operatorname{Coh}(X)$ is a vector bundle, then $f_{*}(V) \in Q \operatorname{Coh}(Y)$ is also a vector bundle. Thus there is an induced map $f_{*}: \operatorname{Iso}(\operatorname{Vect}(X)) \rightarrow$ $I s o(V e c t(Y))$. Since $f_{*}(E \oplus F) \cong f_{*}(E) \oplus f_{*}(F)$, this descends to the Grothendieck group to yield a push-forward homomorphism $\operatorname{tr}_{f}:=f_{*}: K(\operatorname{Vect}(X))^{\oplus} \rightarrow K(\operatorname{Vect}(Y))^{\oplus}$.

If in addition $f$ is étale and $E \in \operatorname{Bil}(X)$ then the trace map $f_{*} \mathcal{O}_{X} \rightarrow \mathcal{O}_{Y}$ can be used to turn $f_{*} E \in V \operatorname{ect}(Y)$ into a bilinear bundle. Indeed we let $b_{f_{*} E}$ be the composite

$$
f_{*} E \otimes f_{*} E \rightarrow f_{*}(E \otimes E) \stackrel{f_{*} b}{\longrightarrow} f_{*} \mathcal{O}_{X} \rightarrow \mathcal{O}_{Y}
$$

Here we have used that $f_{*}$ is right adjoint to a symmetric monoidal functor, so is lax symmetric monoidal. Then as before we obtain $\operatorname{tr}_{f}: K(\operatorname{Bil}(X))^{\oplus} \rightarrow K(\operatorname{Bil}(Y))^{\oplus}$.

The tensor product of bundles turns $I$ so $(V e c t(X))$ and $I s o(\operatorname{Bil}(X))$ into semi-rings, and $K(V e c t(X))^{\oplus}$ and $K(\operatorname{Bil}(X))^{\oplus}$ into rings. However, $t r_{f}$ is not a ring homomorphism: it does not respect multiplication. This can be remedied to some extent by considering a multiplicative version of transfer. In the next two subsections, we explain two constructions of such multiplicative transfers. In the last subsection, we compare the two.

\subsection{The Ferrand norm}

Given a finite locally free ring homomorphism $R \rightarrow S$, in [7] D. Ferrand defined a norm functor $N_{S / R}$ : $S$-Mod $\rightarrow R$-Mod. It is lax symmetric monoidal [7, (N6)] and so preserves algebras, modules over algebras, etc.

We briefly review Ferrand's definition. If $M$ is an $R$-module, we denote by $\underline{M} \in F u n(R$-Alg, Sets $)$ the functor $R^{\prime} \mapsto M \otimes_{R} R^{\prime}$.

Definition 2 (7], 2.2.1). A polynomial law between $R$-modules $M_{1}, M_{2}$ is a natural transformation $\underline{M}_{1} \rightarrow \underline{M}_{2}$.

Recall that if $M$ is a (finitely generated) locally free $R$-module and $\alpha: M \rightarrow M$ is an endomorphism, one can define the determinant $\operatorname{det}(\alpha) \in R$. Indeed if $M$ has rank $n$ then the maximal exterior power $\Lambda^{n}(\alpha): \Lambda^{n} M \rightarrow \Lambda^{n} M$ is an endomorphism of the invertible rank 1 module $\Lambda^{n} M$, so must be given by a multiplication by a (unique) element $\operatorname{det}(\alpha) \in R$. If $M$ is not of constant rank then $R=R_{1} \times R_{2} \times \cdots \times R_{k}$ such that $M=M_{1} \times \cdots \times M_{k}$ with each $M_{i}$ of constant rank, and we let $\operatorname{det}(\alpha)=\left(\operatorname{det}\left(\alpha_{1}\right), \ldots, \operatorname{det}\left(\alpha_{k}\right)\right)$. It is easy to see that this is independent of the decomposition $R=\prod_{i} R_{i}$.

Since $S / R$ is finite locally free by assumption, we thus have the norm map $n_{S / R}: S \rightarrow R, x \mapsto$ $\operatorname{det}(\times x: S \rightarrow S)$. Here $\times x: S \rightarrow S$ denotes the endomorphism $s \mapsto x s$. This defines a polynomial law from $S$ to $R$, because the determinant commutes with base change.

Definition 3 ([7] Definition 3.2.1). If $F \in S$-Mod and $E \in R$-Mod, a norm law from $F$ to $E$ is a polynomial law $\phi$ from $F$ (viewed as an $R$-module) to $E$ such that for every $R$-algebra $R^{\prime}$ and $s \in R^{\prime} \otimes_{R} S, x \in R^{\prime} \otimes_{R} F$ we have $\phi(s x)=n_{R^{\prime} \otimes_{R} S / R^{\prime}}(s) \phi(x)$.

Given $F \in S$-Mod, there is a universal norm law $n_{F}: F \rightarrow N_{S / R}(F)$, in the following sense: if $E \in R$-Mod and $\phi$ is a norm law from $F$ to $E$, then there is a unique $R$-homomorphism $u: N_{S / R}(F) \rightarrow E$ such that $\phi=u \circ n_{F}$ [7. Theorem 3.2.3]. This defines the norm functor.

In geometric language, given a finite locally free morphism $f: X \rightarrow Y$ of affine schemes, we have found a functor $N_{f}: Q \operatorname{Coh}(X) \rightarrow Q \operatorname{Coh}(Y)$. Although we will not need this, let us remark that it is easy to see that the norm construction can be extended to any finite locally free morphism of schemes, affine or not.

\subsection{The Rost norm}

Ferrand's norm functor has nice technical properties (like being defined by a universal property), but it can be difficult to get a hold of computationally. There is an alternative construction due to Rost [22, 3.2]. Again we review the definition briefly.

If $M$ is any $R$-module, we define the $n$-th symmetric power as

$$
\operatorname{Sym}_{R}^{n}(M)=\left(M^{\otimes n}\right)^{\Sigma_{n}},
$$


where $\Sigma_{n}$ is the symmetric group on $n$ letters, acting by permuting the factors of $M^{\otimes n}$, and the superscript means passing to invariants. Note that if $S$ is an $R$-algebra, then so is $S^{\otimes n}$, and $\operatorname{Sym}^{n} S \subset S^{\otimes n}$ is a subalgebra.

Now let $R \rightarrow S$ be locally free of rank $n$. Since $\Lambda_{R}^{n} S$ is a quotient of $S^{\otimes n}$ and $\operatorname{Sym}_{R}^{n} S$ is a subalgebra of $S^{\otimes n}, \Lambda_{R}^{n} S$ is a $\operatorname{Sym}_{R}^{n} S$-module. We thus get a canonical homomorphism

$$
\bar{n}_{S / R}: \operatorname{Sym}_{R}^{n} S \rightarrow \operatorname{End}_{R}\left(\Lambda^{n} S\right) \cong R, x \mapsto\left(\times x: \Lambda^{n} S \rightarrow \Lambda^{n} S\right) .
$$

Here we have used that $\operatorname{End}_{R}\left(\Lambda^{n} S\right) \cong R$ because $\Lambda^{n} S$ is an invertible $R$-module.

Definition 4 (22], 2.3). Let $M$ be an $S$-module. We put

$$
\nu_{S / R}(M)=\operatorname{Sym}_{R}^{n} M \otimes_{\operatorname{Sym}_{R}^{n} S} R,
$$

where the map $\operatorname{Sym}_{R}^{n} S \rightarrow R$ is $\bar{n}_{S / R}$. For more details, see [12.

Reverting to geometric language, for a finite étale morphism of constant rank $f: X \rightarrow Y$ between affine schemes, we have found a functor $\nu_{f}: Q \operatorname{Coh}(X) \rightarrow Q \operatorname{Coh}(Y)$. Again, even though we do not need the extra generality, it is easy to see that this construction extends to any finite étale morphism, not necessarily of constant rank, and not necessarily between affine schemes.

\subsection{Comparison of norms}

We now show that the constructions from the previous two subsections coincide in sufficiently good cases.

Proposition 5. Let $f: X \rightarrow Y$ be a finite étale morphism of schemes. Then for $M \in V$ ect $(X)$ there is a canonical isomorphism $\nu_{f}(M) \cong N_{f}(M)$.

Proof. Since the isomorphisms are canonical, i.e. compatible with base change in $Y$, they can be glued in open covers. Thus we may assume that $Y=\operatorname{Spec}(R), X=\operatorname{Spec}(S)$ and $R \rightarrow S$ is finite étale of rank $n$. Then the vector bundle $M$ corresponds to a locally free $S$-module which we still denote by $M$.

There is an evident map

$$
\alpha_{M}: M \rightarrow \nu_{S / R}(M), m \mapsto(m \otimes m \otimes \cdots \otimes m) \otimes 1 .
$$

To be clear, this is not a homomorphism. I claim that it defines a norm law. We first need to show that it is a polynomial law. Hence if $R_{1} \rightarrow R_{2}$ is a homomorphism of $R$-algebras, we need to show that the following square commutes:

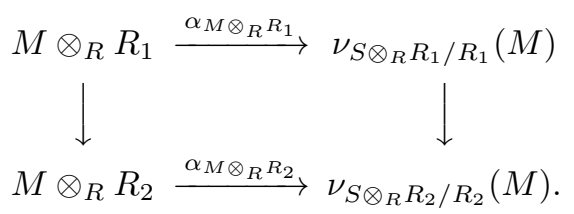

Here the left vertical map is the induced one. We have $\operatorname{Sym}_{R^{\prime}}^{n}\left(M \otimes_{R} R^{\prime}\right) \cong \operatorname{Sym}_{R}^{n}(M) \otimes_{R} R^{\prime}$ [12, Korollar 2.3.2] and consequently $\nu_{S \otimes_{R} R^{\prime} / R^{\prime}}(M) \cong \nu_{S / R}(M) \otimes_{R} R^{\prime}$. The right vertical map is the one induced by this isomorphism. Checking commutativity is then routine. The proof that this is a norm law boils down to the claim that for $s \in S$ we have $\nu_{S / R}(s \otimes s \otimes \cdots \otimes s)=n_{S / R}(s) \in R$. This follows from [12, Korollar 4.1.2] and [7, (N1)].

By universality of the norm law $M \rightarrow N_{S / R}(M)$ there exists a unique $R$-linear map $\beta_{M}: N_{S / R}(M) \rightarrow$ $\nu_{S / R}(M)$ such that the composite $M \rightarrow N_{S / R}(M) \rightarrow \nu_{S / R}(M)$ is $\alpha_{M}$. We claim that $\beta_{M}$ is an isomorphism. To see this, we may perform a faithfully flat base change and assume that $S \cong R^{d}$. But then $M \cong \prod_{i=1}^{d} M_{i}$ and $N(M) \cong \bigotimes_{i} M_{i} \cong \nu(M)$ [7. Lemme 3.2.4] [12, Satz 4.3.2] (this is where we need $M$ locally free). Moreover $n_{M}: M \rightarrow N(M)$ is given by $\left(m_{1}, \ldots, m_{d}\right) \mapsto\left(m_{1} \otimes m_{2} \otimes \ldots m_{d}\right)$, c.f. [7, Exemple 3.2.2.c)]. It follows from uniqueness of $\beta_{M}$ that $\beta_{M}$ is indeed the canonical isomorphism. This concludes the proof.

In the last paragraph of the above proof we have also established the following result:

Proposition 6. Let $f: X \coprod X \coprod \cdots \coprod X \rightarrow X$ be the fold map of an $n$-fold coproduct. Then $N_{f}:$ $V \operatorname{ect}(X \coprod \cdots \coprod X) \cong V \operatorname{ect}(X)^{n} \rightarrow \operatorname{Vect}(X)$ is given by $\left(E_{1}, \ldots, E_{n}\right) \mapsto E_{1} \otimes \cdots \otimes E_{n}$.

If $f: X \rightarrow Y$ is finite étale and $E \in V e c t(X)$, then $\nu_{f}(E) \in V e c t(Y)$, as follows for example from [12, Satz 4.3.2]. The functor $\nu_{f}=N_{f}: \operatorname{Vect}(X) \rightarrow V e c t(Y)$ is symmetric monoidal [12, Korollar 4.3.4]. Since $N_{f}\left(\mathcal{O}_{X}\right)=\mathcal{O}_{Y}$ it follows easily that the functor preserves bilinear bundles, cf. also 12, Korollar 4.2.7]. We have thus found $N_{f}: I s o(V e c t(X)) \rightarrow \operatorname{Iso}(V e c t(Y))$ and $N_{f}: \operatorname{Iso}(\operatorname{Bil}(X)) \rightarrow I s o(\operatorname{Bil}(Y))$, and these are homomorphisms of multiplicative monoids. 


\section{$3 G W$ as a Tambara functor}

Recall that if $f: X \rightarrow Y$ is any morphism of schemes, then the pullback $f^{*}: Q \operatorname{Coh}(Y) \rightarrow Q \operatorname{Coh}(X)$ induces $f^{*}: \operatorname{Iso}(\operatorname{Vect}(Y)) \rightarrow \operatorname{Iso}(\operatorname{Vect}(X))$ and $f^{*}: \operatorname{Iso}(\operatorname{Bil}(Y)) \rightarrow \operatorname{Iso}(\operatorname{Bil}(X))$, and these are homomorphisms of semi-rings, i.e. respect both the multiplicative and additive structure. Suppose that $f$ is finite étale. Then as we have seen in the previous section, we can construct $N_{f}: \operatorname{Iso}(\operatorname{Bil}(X)) \rightarrow \operatorname{Iso}(\operatorname{Bil}(Y))$, which is a homomorphism of multiplicative monoids, and $t r_{f}: \operatorname{Iso}(\operatorname{Bil}(X)) \rightarrow \operatorname{Iso}(\operatorname{Bil}(Y))$, which is a homomorphism of additive monoids. In this section we will explain the compatibilities between these three operations. It turns out that Tambara 27] has studied precisely this kind of situation. As a consequence, we will be able to deduce that $N_{f}: \operatorname{Iso}(\operatorname{Bil}(X)) \rightarrow \operatorname{Iso}(\operatorname{Bil}(Y))$ extends in a canonical way to $N_{f}: K(\operatorname{Bil}(X))^{\oplus} \rightarrow K(\operatorname{Bil}(Y))^{\oplus}$, and that this extension has many desirable properties, such as a base change formula. This extension has been produced before in a different way by M. Rost [22].

We write Fét for the category of all schemes, with morphisms the finite étale morphisms. For a scheme $S$, we let Fét/S denote the usual slice category. Recall that any morphism between schemes which are finite étale over $S$ is automatically finite étale, so $F e ́ t / S$ is also the category of finite étale schemes over $S$, with all morphisms of schemes allowed.

If $f: X \rightarrow Y \in F e ́ t / S$ is a morphism, then we get as usual a functor $f^{*}: F e ́ t / Y \rightarrow F e ́ t / X$. This functor has a right adjoint $f_{*}$ which is in fact isomorphic to Weil restriction along $f$. To see this, it suffices to show that if $T \in F e ́ t / X$ then the Weil restriction $R_{X / Y}(T) \in S c h / Y$ is finite étale. This is clear from infinitesimal lifting criteria; see [5, Proposition 7.5.5].

The following definitions are modeled in an evident way on [14, Definition 1.4.1].

Definition 7. Given morphisms $A \stackrel{q}{\rightarrow} X \stackrel{f}{\rightarrow} Y$ in Fét/S, we can build the following commutative diagram (in Fét/Y or Fét/S) called the exponential diagram generated by $A \rightarrow X \rightarrow Y$ :

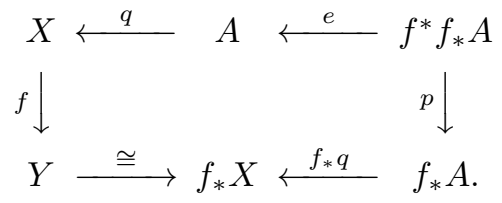

Here $f_{*}$ denotes the functor $f_{*}: F e ́ t / X \rightarrow F e ́ t / Y$. Being a right adjoint it preserves final objects, whence the claimed isomorphism. The left adjoint is $f^{*}:$ Fét/Y $\rightarrow$ Fét/X. The map $e: f^{*} f_{*} A \rightarrow A$ is the counit of this adjunction. The functor $f^{*}$ also has a left adjoint $f_{\#}$, and $p: f_{\#} f^{*} f_{*} A \rightarrow f_{*} A$ is the counit of this further adjunction.

As in the exponential diagram above, we usually will not distinguish between $f_{\#} T$ and $T$. Indeed $f_{\#} T$ is just " $T$ viewed as a $Y$-scheme in the canonical way", so this should not cause confusion.

Definition 8. A Tambara functor $F$ over $S$ consists of the following data: for each $X \in F$ ét $/ S$ a semiring $F(X)$, together with for each $f: X \rightarrow Y \in F$ ét/S three maps $f^{*}: F(Y) \rightarrow F(X), \operatorname{tr}_{f}: F(X) \rightarrow$ $F(Y)$ and $N_{f}: F(X) \rightarrow F(Y)$. This data is required to satisfy the following conditions:

1. $F(X \amalg Y) \cong F(X) \times F(Y)$, via the canonical map

2. $f^{*}$ is a homomorphism, $t r_{f}$ is a homomorphism of additive monoids, and $N_{f}$ is a homomorphism of multiplicative monoids

3. $f^{*}, t r_{f}, N_{f}$ are functorial in $f$ (i.e. $(f g)^{*}=g^{*} f^{*}$, id ${ }^{*}=\mathrm{id}$, and so on)

4. Transfer and norm commute with base change, in the sense that given a cartesian square in $F e ́ t / S$

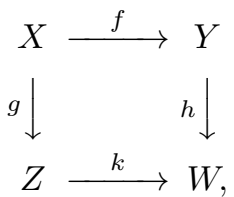

the following square commutes

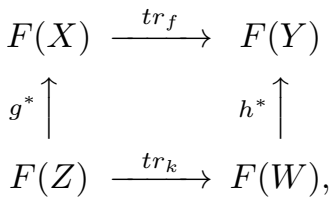

and similarly with $N$ in place of $t r$. 
5. Given morphisms $Z \stackrel{q}{\rightarrow} X \stackrel{f}{\rightarrow} Y$ (in Fét/S), the following diagram induced by the associated exponential diagram commutes:

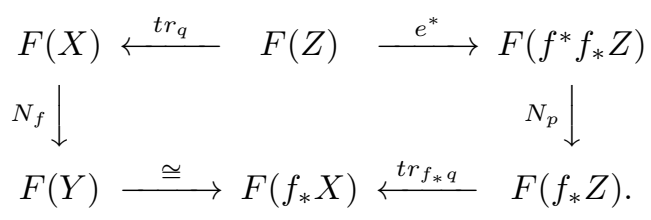

Remark 9. Conditions 2,3 and 4 above imply that if $f: X^{n} \rightarrow X$ is the fold map, then $\operatorname{tr}_{f}: F\left(X \amalg^{n}\right) \cong$ $F(X)^{n} \rightarrow F(X)$ is just addition in $F(X)$, and similarly $N_{f}$ is multiplication.

Remark 10. Condition 5 above should be seen as a generalized distributivity law. Indeed if all finite étale morphisms occurring are fold maps (i.e. are split), then this condition precisely expresses that multiplication distributes over addition in the semi-ring $F(X)$. As a consequence, we see that a Tambara functor can alternatively be defined as a presheaf of sets (not semi-rings) with norms and transfers, satisfying conditions $1,3,4,5$; then there is a unique structure of a semi-ring on each $F(X)$ such that 2 is satisfied. We will not use this observation.

Proposition 11. The assignments $X \mapsto I s o(\operatorname{Vect}(X))$ and $X \mapsto I \operatorname{so}(\operatorname{Bil}(X))$ define Tambara functors on $S$.

Proof. We need to verify the axioms. Condition 1 is obvious, condition 2 is obvious for $f^{*}$. It holds for $t r_{f}$ because $f_{*}$ preserves direct sums, and it holds for $N_{f}$ because the norm functor is symmetric monoidal, see for example [12, Satz 4.4.4]. Alternatively, the argument of Remark 10 reduces this to Proposition 6. Condition 3 for $f^{*}$ is well-known, for $t r_{f}$ it follows from functoriality of $f_{*}$, and for $N_{f}$ it follows from functoriality of the norm [7, Proposition 3.2.5 b) and c)]. Similarly condition 4 follows from the base change formula for $f_{*}$ (well-known) and for the norm [7, (N2)].

We thus need to establish condition 5 . We do this in some detail; this style of argument can also be used to make more precise our sketches for conditions 1-4. Given morphisms $Z \stackrel{q}{\rightarrow} X \stackrel{f}{\rightarrow} Y$ in Fét/S and $a \in I \operatorname{so}(\operatorname{Vect}(Z))$ (respectively $\bar{a} \in I \operatorname{so}(\operatorname{Bil}(Z))$ ) we need to show that $N_{f} t r_{q}(a)=\operatorname{tr}_{f_{*} q} N_{p} e^{*}(a)$, and similarly for $\bar{a}$. In order to do this, for $E \in V \operatorname{ect}(Z)$, we shall (1) exhibit a natural morphism $N_{f} q_{*} E \rightarrow\left(f_{*} q\right)_{*} N_{p} E$. Natural here means natural in $E$ and also in $Y$, i.e. compatible with base change. Then we shall (2) verify that this morphism is an isomorphism, and that if $E \in B i l(Z)$, then the morphism respects the bilinear structure. Note that problem (1) is Zariski-local on $X$, and problem (2) is even local in the faithfully flat topology. Consequently to solve (1) we may assume that $Y$ is affine, say $Y=\operatorname{Spec}(A)$, and for (2) we may even assume that $X=\operatorname{Spec}\left(A^{n}\right)$.

Step 1. We know that Weil restriction is isomorphic to the norm construction [7, Proposition 6.2.2]. We are thus given the following diagram of commutative rings (with all maps finite étale homomorphisms)

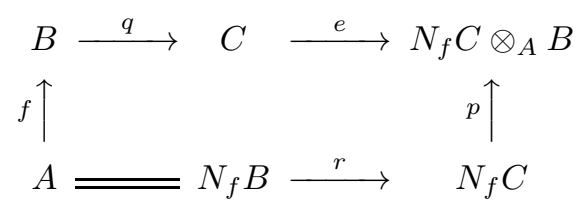

together with a locally free module $M$ on $C$, and we need to exhibit a functorial morphism of $A$-modules

$$
N_{f} M \rightarrow N_{p}\left(M \otimes_{C}\left(N_{f} C \otimes_{A} B\right)\right)
$$

Recall that on the level of modules, the pushforward operation just coincides with forgetting some of the module structure; this is why there are no transfer operators in the above formula.

We shall construct a norm law $B$-Mod $\ni M \rightarrow N_{p}\left(M^{\prime}\right) \in A$-Mod, where $M^{\prime}:=M \otimes_{C}\left(N_{f} C \otimes_{A} B\right)$. By universality of the norm law $M \rightarrow N_{f} M$, this induces a homomorphism $N_{f} M \rightarrow N_{p} M^{\prime}$ as desired.

To do this, consider the composite $\phi: M \rightarrow M^{\prime} \rightarrow N_{p}\left(M^{\prime}\right)$, where $M \rightarrow M^{\prime}$ is $m \mapsto m \otimes 1$ and $M^{\prime} \rightarrow N_{p}\left(M^{\prime}\right)$ is the norm map. This defines a polynomial law over $A$ because the entire diagram is functorial under base change in $A$, which follows from condition 4 (which we have already established). To see that this defines a norm law (see Definition [3) we need to check that for $b \in B$ and $m \in M$ we have

$$
\phi(b m)=n_{f}(b) \phi(m)
$$


Here for any $B$-module $L$, we write $n_{f}: L \rightarrow N_{f} L$ for the universal norm law. By definition we have $\phi(m)=n_{p}(m \otimes 1)$ and hence $\phi(b m)=n_{p}(e(q(b))) \cdot \phi(m)$, since $n_{p}$ is a norm law. We thus need to show that

$$
n_{p}(e(q(b)))=r\left(n_{f}(b)\right) .
$$

Since all our modules are locally free, hence flat, we may check this after any base change along an injective ring homomorphism, e.g. a faithfully flat one.

We may thus assume that $B=A^{d}$ and hence that $C=\prod_{i=1}^{d} C_{i}$. Then $N_{f} C=\bigotimes_{i=1}^{d} C_{i}$. The canonical map $e: C \rightarrow N_{f} C \otimes_{A} B=\left(N_{f} C\right)^{d}$ is given by

$$
\left(c_{1}, \ldots, c_{d}\right) \mapsto\left(c_{1} \otimes 1 \otimes \ldots, 1 \otimes c_{2} \otimes 1 \otimes \ldots, \ldots, 1 \otimes 1 \otimes \cdots \otimes 1 \otimes c_{d}\right),
$$

as one checks by verifying the universal property of a unit of adjunction. The norm maps are $n_{f}: B=$ $A^{d} \rightarrow A,\left(a_{1}, \ldots, a_{d}\right) \mapsto a_{1} \ldots a_{d}$ and similarly for $n_{p}$. Then $n_{p} e q=r n_{f}: B \rightarrow N_{f} C$ follows by direct computation.

Step 2. We remain in the situation above, i.e. we have $A \rightarrow B \rightarrow C$ with $B=A^{d}$ and $C=\prod_{i} C_{i}$. Moreover we have a $B$-module $M$ and canonical morphism $N_{f} M \rightarrow N_{p} M^{\prime}$. We need to check that this is an isomorphism, respecting bilinear structures if present.

We first check that the morphism is an isomorphism. We have $M=\prod_{i=1}^{d} M_{i}$, where $M_{i}$ is a $C_{i^{-}}$ module. Then $N_{f} M=\bigotimes_{i=1}^{d} M_{i}$, where the tensor product is over $A$. In contrast, $M^{\prime}=\prod_{i=1}^{d}\left(M_{i} \otimes_{C_{i}}\right.$ $N_{f} C$ ), and $N_{p} M^{\prime}$ is the tensor product of these terms over $N_{f} C$. Since $M_{1} \otimes C_{1} N_{f} C=M_{1} \otimes C_{2} \otimes \cdots \otimes C_{d}$ and so on, it is easy to see by direct computation that $N_{f} M \rightarrow N_{p} M^{\prime}$ is an isomorphism.

Similarly, giving a bilinear form $\psi: M \otimes_{B} M \rightarrow B$ is equivalent to giving $\psi_{i}: M_{i} \otimes M_{i} \rightarrow A$. The induced bilinear form on $N_{f} M=\bigotimes_{i} M_{i}$ is $N_{f}(\psi)\left(m_{1} \otimes \cdots \otimes m_{d}\right)=\prod_{i} \psi_{i}\left(m_{i}\right)$. Arguing similarly for $M^{\prime}$, it is easy to check by direct computation that $N_{f} M \rightarrow N_{p} M^{\prime}$ is compatible with the bilinear forms. This concludes the proof.

Remark 12. We can formally invert the sum operation in $I$ so $(V e c t(X))$ and then obtain the Grothendieck ring $K(V e c t(X))^{\oplus}$. It is a priori not at all clear that the norm map $N_{f}: I \operatorname{so}(V e c t(X)) \rightarrow I \operatorname{so}(V e c t(Y))$ induces a map $K(V e c t(X))^{\oplus} \rightarrow K(V e c t(Y))^{\oplus}$. The main point of 22 is that this indeed works, and the proof is by showing that the norm maps are polynomial (in a sense that is a priori stronger than the definition we have used so far) and then showing that polynomial maps descend to Grothendieck groups.

It is also possible to deduce this fact from our proposition. Indeed, any Tambara functor may be "additively completed" (i.e. one may pass to the Grothendieck ring) 27, Theorem 6.1].

The same discussion can be repeated with $\operatorname{Bil}(X)$ in place of $V e c t(X)$.

Using either of the above mentioned results, we obtain the following.

Corollary 13. The assignments $X \mapsto K(\operatorname{Vect}(X))^{\oplus}$ and $X \mapsto K(\operatorname{Bil}(X))^{\oplus}$ define Tambara functors on $S$.

\section{The $G W$-module structure on $G W^{\times}$}

In this section we begin in earnest the program sketched in the introduction: using the multiplicative transfers studied in the previous two sections, we turn the group of units in $G W(k)^{\times}$into a module over $G W(k)$, where $k$ is a field.

We consider the Tambara functor $K(\operatorname{Bil}(\bullet))=G W(\bullet)$ on Fét $/ k$. If $A / k$ is a finite étale algebra, we write $N_{A / k}$ and $t r_{A / k}$ for the multiplicative and additive transfer, and $\left.x \mapsto x\right|_{A}$ for the restriction. We put $\operatorname{tr}(A):=\operatorname{tr}_{A / k}(1)$.

Recall the dimension homomorphism $\operatorname{dim}: G W(A) \rightarrow \mathbb{Z}^{d}$, where $d$ is the number of connected components of $\operatorname{Spec}(A)$. Its kernel is called the fundamental ideal and denoted $I(A)$. Note that $I(A \times$ $B)=I(A) \times I(B)$. Since $G W(A)$ is a ring it has a subset of units $G W^{\times}(A)$. This is a group where the operation is multiplication in the Grothendieck-Witt ring. We put for $n \geq 0$

$$
F_{n} G W^{\times}(A)=\left\{x \in G W^{\times}(A) \mid x \equiv 1 \quad\left(\bmod I^{n}(A)\right)\right\} .
$$

In other words, $F_{n} G W^{\times}(A)=\left(1+I^{n}(A)\right) \cap G W^{\times}(A)$. We will make good use of the map

$$
\alpha_{n}: F_{n} G W^{\times}(A) \rightarrow I^{n}(A) / I^{2 n}(A), x \mapsto x-1 .
$$


Note that it is a homomorphism, where we use the multiplicative group structure on the left and the additive structure on the right. In fact $I^{n}(A) / I^{2 n}(A)$ is the largest quotient $I^{n}(A) / J$ of $I^{n}(A)$ such that the map $\left(1+I^{n}(A), \times\right) \rightarrow\left(I^{n}(A) / J,+\right), x \mapsto x-1$ is a homomorphism of monoids.

Since we shall use it all the time, let us make explicit the following well-known fact.

Lemma 14 (Arason [1], Satz 3.3). Let $A / k$ be a finite étale algebra. Then $\operatorname{tr}_{A / k}\left(I^{n}(A)\right) \subset I^{n}(k)$.

For the purpose of this section, we will always view $I(k)$ as an ideal of $G W(k)$. We thus put $I^{0}(k):=G W(k)$, and not $I^{0}(k)=W(k)$ as may be more customary.

The following well-known result is very useful for computations.

Lemma 15. Let $l / k$ be an algebraic field extension of odd degree. In other words $l / k$ is algebraic, and if $l / l_{0} / k$ is a subextension with $l_{0} / k$ finite, then $\left[l_{0}: k\right]$ is odd. For $0 \leq n \leq \infty$, the restriction map

$$
I^{n}(k) / I^{m}(k) \rightarrow I^{n}(l) / I^{m}(l)
$$

is injective. Here we put $I^{\infty}(k):=0$.

Proof. In the proof we shall use transfers along finite extensions which are not separable in general; these are also known as Scharlau transfers. The only difference is that there need not be a unique transfer, but rather there will be a family of transfers differing by multiplication by a one-dimensional form. The choice will not matter to us, since we use only the most basic properties. The most subtle one is Lemma 14. which holds in this generality (and is in fact stated in this generality by Arason). In this text we shall only ever apply Lemma 15 to separable extensions anyway.

By continuity (see Corollary 51 in Appendix B), we may assume that $l / k$ is a finite extension.

Let us first show that $I^{n}(k) / I^{n+1}(k) \rightarrow I^{n}(l) / I^{n+1}(l)$ is injective. Since transfer preserves $I^{n}$ by Lemma 14, we get a well-defined map $\operatorname{tr}: I^{n}(l) / I^{n+1}(l) \rightarrow I^{n}(k) / I^{n+1}(k)$. It suffices to show that the composite $\alpha: I^{n}(k) / I^{n+1}(k) \rightarrow I^{n}(l) / I^{n+1}(l) \rightarrow I^{n}(k) / I^{n+1}(k)$ is injective. By the projection formula (see [13, Theorem VII.1.3] for the inseparable case), $\alpha$ is given by multiplication by an element $t \in G W(k)$ of dimension $[l: k]$. In other words $t \equiv[l: k](\bmod I)$ and consequently $\alpha$ is given by multiplication by $[l: k]$. Since $2 I(k) \subset I^{2}(k)$, i.e. $I^{n}(k) / I^{n+1}(k)$ is an $\mathbb{F}_{2}$-vector space, multiplication by the odd integer $[l: k]$ is injective.

If $0 \leq n \leq m<\infty$ then $I^{n}(k) / I^{m}(k) \rightarrow I^{n}(l) / I^{m}(l)$ is a morphism of finitely filtered abelian groups which is injective on the subquotients (by what we have just shown), hence injective. The case $m=\infty$ follows from the fact that $\cap_{m} I^{m}(k)=0$ [24, Corollary 4.5.7], or directly from Springer's theorem [24, Corollary 2.5.4].

By a degree 2 extension $A$ of a field $k$ of characteristic different from 2 we shall always mean a degree 2 étale extension; this is either a quadratic field extension of $k$, or the extension $A=k \times k$. Such an extension has a canonical automorphism denoted by $x \mapsto \bar{x}$. If $A / k$ is a quadratic extension then $x \mapsto \bar{x}$ is the non-trivial Galois automorphism. If $A=k \times k$ then we put $\overline{(x, y)}:=(y, x)$. Then for any $A / k$ of degree 2, the formulas $\operatorname{tr}_{A / k}(x)=x+\bar{x}$ and $N_{A / k}(x)=x \bar{x}$ are correct for all $x \in A$, and $k \hookrightarrow A$ consists precisely of the invariants of $x \mapsto \bar{x}$. We then have the following slight extension of a result of Wittkop we shall use extensively.

Lemma 16 (Wittkop [29]). Let $\operatorname{char}(k) \neq 2, A / k$ of degree 2 and $x, y \in G W(A)$. Then

$$
N_{A / k}(x+y)=N_{A / k}(x)+N_{A / k}(y)+t r_{A / k}(x \bar{y}) .
$$

Proof. If $A / k$ is quadratic, then this is [29, Satz 2.5(ii)]. If $A=k \times k$ then $x=\left(x_{1}, x_{2}\right), y=\left(y_{1}, y_{2}\right)$ and by Proposition 6 (and its additive analogue, cf. Remark 9) we have

$$
N(x+y)=\left(x_{1}+y_{1}\right)\left(x_{2}+y_{2}\right)=x_{1} x_{2}+y_{1} y_{2}+\left(x_{1} y_{2}+x_{2} y_{1}\right)=N(x)+N(y)+\operatorname{tr}(x \bar{y}) .
$$

The following is a very basic result. Its proof illustrates nicely how to use Lemmas 15 and 16. We give many details because we shall re-use the technique several times. Note in particular how testing equalities between elements constructed using norms and transfers is reduced to the case of degree 2 extensions.

Proposition 17. Let $A / k$ be a finite étale algebra. 
(i) If $A / k$ is of degree $p$, we have $N_{A / k}\left(I^{n}(A)\right) \subset I^{n p}(k)$.

(ii) We have $N_{A / k}\left(F_{n} G W^{\times}(A)\right) \subset F_{n} G W^{\times}(k)$.

(iii) Recall the map $\alpha_{n}$ from equation (11). In the situation of (ii), moreover the following diagram commutes:

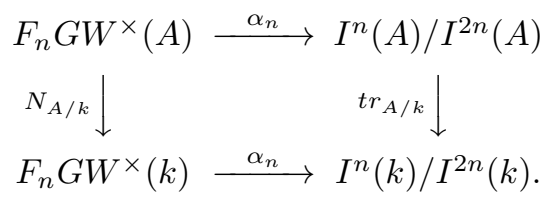

Proof. Let $l / k$ be a finite Galois extension such that all residue fields of $A$ embed in $l, H$ a 2-Sylow subgroup of $G=\operatorname{Gal}(l / k)$ and $k^{\prime}=l^{H} / k$ the associated field extension. Let $A^{\prime}:=A \otimes_{k} k^{\prime}$ denote the scalar extension. By Corollary 13 we have a commutative diagram

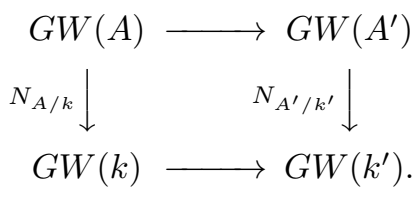

For (i), we wish to show that the composite $I^{n}(A) \rightarrow G W(A) \stackrel{N_{A / k}}{\longrightarrow} G W(k) \rightarrow G W(k) / I^{n p}(k)$ is zero. We stress that it is not a homomorphism; this will not matter. Since $\left[k^{\prime}: k\right]=|G / H|$ is odd, by Lemma 15 we know that $G W(k) / I^{n p}(k) \rightarrow G W\left(k^{\prime}\right) / I^{n p}\left(k^{\prime}\right)$ is injective. Hence consulting the commutative diagram, we find that we may assume (replacing $k$ by $k^{\prime}$ ) that $G$ is a 2 -group.

I claim that in this case, for any subextension $k \subset l^{\prime} \subset l$ there exist intermediate extensions $k=$ $k_{0} \subset k_{1} \subset \cdots \subset k_{p}=l^{\prime}$ such that $\left[k_{i+1}: k_{i}\right]=2$ for all $i$. To see this, we may assume by induction that $k \subset l^{\prime}$ has no intermediate extensions, i.e. $\operatorname{Gal}\left(l / l^{\prime}\right) \subset \operatorname{Gal}(l / k)$ is a proper maximal subgroup. Any such subgroup is of index 2 [23, Theorem 4.6], so the claim is proved.

Now $A=l_{1} \times \cdots \times l_{r}$, where by construction each $l_{i}$ embeds into $l$. Suppose that we can prove (i) for each of the $l_{i} / k$. Then if $x=\left(x_{1}, \ldots, x_{r}\right) \in I^{n}(A)$ we get

$$
N_{A / k}(x)=\prod_{i} N_{l_{i} / k}\left(x_{i}\right) \in \prod_{i} I^{\left[l_{i}: k\right] n}(k) \subset I^{n p}(k),
$$

since $p=\sum_{i}\left[l_{i}: k\right]=[A: k]$. We thus need only prove the result for $A=l$ a field. In this case we use the claim to factor $k \rightarrow A=l$ as $k \rightarrow k_{1} \rightarrow \cdots \rightarrow k_{q}=l$, with $\left[k_{i+1}: k_{i}\right]=2$. Using transitivity of the norm we reduce to the case $[l: k]=2$.

If $x, y \in I^{n}(l)$ with $N_{l / k}(x), N_{l / k}(y) \in I^{2 n}(k)$ then $N_{l / k}(x+y)=N_{l / k}(x)+N_{l / k}(y)+\operatorname{tr}_{l / k}(x \bar{y}) \in$ $I^{2 n}(k)$, by Lemmas 16 and 14. Note that the conjugation $y \mapsto \bar{y}$ preserves $I^{n}(l)$. Moreover in this situation we have $N_{l / k}(-x)=N_{l / k}(-1) N_{l / k}(x) \in I^{2 n}(k)$. It is thus enough to show that $N_{l / k}(x) \in I^{2 n}(k)$ for an additive generating set of elements $x$ of $I^{n}(l)$. This follows from 29, Lemma 1.56 and Satz 2.16 (ii),(iv)]. We have thus proved (i).

The proof of (ii) proceeds similarly, using the composite $F_{n} G W^{\times}(A) \stackrel{N_{A / k}}{\longrightarrow} G W^{\times}(k) \stackrel{x \mapsto x-1}{\longrightarrow}$ $G W(k) / I^{2 n}(k)$. We may assume that there is a Galois extension $l / k$ with $G a l(l / k)$ a 2 -group such that each residue field of $A$ embeds into $l$. We factor $k \rightarrow A$ as $k \rightarrow k^{r} \rightarrow \prod_{i} l_{i}$; this allows us to reduce to $A$ a field, which using the claim we may assume is of degree 2 over $k$, or $A=k^{r}$. But $N_{k^{r} / k}\left(x_{1}, \ldots, x_{r}\right)=\prod_{i} x_{i}=N_{k^{2} / k}\left(x_{1}, N_{k^{2} / k}\left(\ldots N_{k^{2} / k}\left(x_{r-1}, x_{r}\right) \ldots\right)\right)$, so the case $A=k^{r}$ reduces to $A=k^{2}$. In other words we have reduced to the case of a degree 2 extension.

Thus we need to show that if $A / k$ is of degree 2 and $1+x \in F_{n} G W^{\times}(A)$, then $N_{A / k}(1+x) \in$ $F_{n} G W^{\times}(k)$. Since the norm is multiplicative it is clear that $N(1+x)$ is invertible, so it suffices to show that $N_{A / k}(1+x) \in 1+I^{n}(k)$. But $N_{A / k}(1+x)=1+t r_{A / k}(x)+N_{A / k}(x)$ by Lemma 16] again, and $\operatorname{tr}_{A / k}(x), N_{A / k}(x) \in I^{n}(k)$ by Lemma 14 and part (i).

To prove part (iii), we may again assume that $A / k$ is of degree 2 . Let $1+x \in F_{n} G W^{\times}(A)$. Then $N_{A / k}(1+x)=1+N_{A / k}(x)+t r_{A / k}(x)$ by Lemma 16 once more. Since $N_{A / k}(x) \in I^{2 n}(k)$ by (i) we have

$$
\alpha_{n}\left(N_{A / k}(1+x)\right)=\left[N_{A / k}(x)+\operatorname{tr}_{A / k}(x)\right]=\left[\operatorname{tr}_{A / k}(x)\right]=\operatorname{tr}_{A / k}\left(\alpha_{n}(1+x)\right),
$$

which concludes the proof. 
Remark 18. The above proof can be made more uniform by using locally constant integers. Namely, if $p$ is a function on $\operatorname{Spec}(A)=\coprod_{i} \operatorname{Spec}\left(l_{i}\right)$ with values in $\mathbb{N}$ (i.e. a non-negative locally constant integer on $\operatorname{Spec}(A))$, then we put $I^{p}(A)=\prod_{i} I^{p\left(\operatorname{Spec}\left(l_{i}\right)\right)}\left(l_{i}\right) \subset G W(A)$. Now suppose that $f: A_{1} \rightarrow A_{2}$ is a finite étale morphism and $p$ is a locally constant integer on $\operatorname{Spec}\left(A_{2}\right)$. We define a locally constant integer $\operatorname{tr}_{A_{2} / A_{1}}(p)$ on $\operatorname{Spec}\left(A_{1}\right)$ by

$$
\operatorname{tr}_{A_{2} / A_{1}}(p)(x)=\sum_{y \in \operatorname{Spec}(f)^{-1}(\{x\})}[k(y): k(x)] p(y) .
$$

Note that if $A / k$ is of degree $p$ and we view $n \in \mathbb{Z}$ as a constant function on $\operatorname{Spec}(A)$, then $\operatorname{tr}_{A / k}(n)=n p$.

Now suppose that $A_{1} \rightarrow A_{2}$ is a finite étale morphism of finite étale $k$-algebras, and $n$ is a locally constant integer on $\operatorname{Spec}\left(A_{2}\right)$. One may show that then

$$
N_{A_{2} / A_{1}}\left(I^{n}\left(A_{2}\right)\right) \subset I^{t r_{A_{2} / A_{1}}(n)}\left(A_{1}\right) .
$$

This statement includes statement (i) above as a special case. It is not difficult to use the same ideas as in the proof of (ii) to reduce this more general statement to the case of a degree 2 extension. We chose to give an ad hoc argument to avoid the complication of locally constant integers. Similar remarks apply at various points in the sequel.

Remark 19. For $n=0$ the statement (iii) of the proposition is not useful. Instead, I claim that the following diagram commutes:

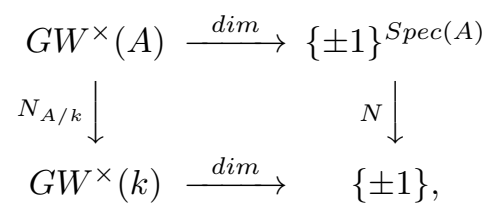

where $N$ on the right hand side is defined as follows. Suppose $A=l_{1} \times \cdots \times l_{r}$, with each $l_{i}$ a field, and $f \in\{ \pm 1\}^{\operatorname{Spec}(A)}$. Then $N(f)=\prod_{i} f\left(\operatorname{Spec}\left(l_{i}\right)\right)^{\left[l_{i}: k\right]}$. Note that $\operatorname{Spec}(A) \mapsto\{ \pm 1\}^{\operatorname{Spec}(A)}$ is a presheaf, $\{ \pm 1\}^{\operatorname{Spec}(k)}=\{ \pm 1\}$ for any field $k$, and that the operation $N$ defined above satisfies the base change formula. In particular, for any field extension $l / k$, the following diagram commutes:

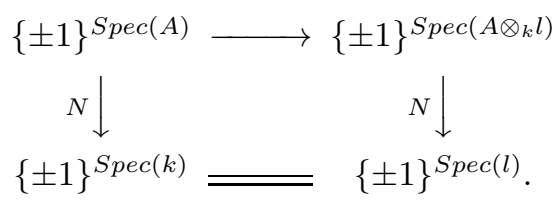

Since $G W$ also satisfies the base change formula, in order to prove the claim we may thus assume that $k$ is algebraically closed. In this case $\operatorname{dim}: G W(A) \rightarrow \mathbb{Z}^{\operatorname{Spec}(A)}$ is an isomorphism, and the diagram commutes by Proposition 6 .

The following observation will allow us to turn the norm maps into a $G W$-module structure.

Lemma 20. Let $A / k, B / k$ be finite étale algebras and $x \in G W(k)$. If $\operatorname{tr}(A)=\operatorname{tr}(B) \in G W(k)$ then $N_{A / k}\left(\left.x\right|_{A}\right)=N_{B / k}\left(\left.x\right|_{B}\right) \in G W(k)$.

Proof. Since $\operatorname{tr}(A)=\operatorname{tr}(B)$ we must have $[A: k]=[B: k]$. It follows from Remark[19 that $\operatorname{dim}\left(N_{A / k}\left(\left.x\right|_{A}\right)\right)=$ $\operatorname{dim}(x)^{[A: k]}=\operatorname{dim}\left(N_{B / k}\left(\left.x\right|_{B}\right)\right)$. It is thus enough to show that $\left[N_{A / k}\left(\left.x\right|_{A}\right)\right]=\left[N_{B / k}\left(\left.x\right|_{B}\right)\right] \in W(k)$. For this we use Serre's splitting principle. For $l / k$ some field extension and $E \in E t_{n}(l)$ an étale algebra of degree $n:=[A: k]$, let $\phi(E)=\left[N_{E / l}\left(\left.x\right|_{E}\right)\right] \in W(l)$. This defines an invariant in the sense of [8, Definition 1.1], by the base change formula. It follows from [8, Theorem 29.2] that there exist $x_{0}, \ldots, x_{n} \in W(k)$ (depending only on $x$, not on $E$ ) such that

$$
\phi(E)=x_{0}+x_{1}\left[\lambda^{1}(\operatorname{tr}(E))\right]+\cdots+x_{n}\left[\lambda^{n}(\operatorname{tr}(E))\right]
$$

The claim follows.

Remark 21. If $E / k$ is a quadratic extension, then it follows from [29, Satz 2.10] that

$$
\left[N_{E / k}\left(\left.x\right|_{E}\right)\right]=\left[x^{2}-2 \lambda^{2}(x)\right]+\left[\lambda^{2}(x)\right] \cdot[\operatorname{tr}(E)] \in W(k),
$$

where $\lambda^{2}(x)$ refers to the canonical $\lambda$-ring structure on $G W(k)$. In other words, in the last step of the proof, if $n=2$, one may take $x_{0}=\left[x^{2}-2 \lambda^{2}(x)\right]$ and $x_{1}=\left[\lambda^{2}(x)\right]\left(\right.$ and $\left.x_{2}=0\right)$. 
If $l=k(\sqrt{a})$ then $\operatorname{tr}(l)=\langle 2\rangle+\langle 2 a\rangle[29$, Lemma 2.3 (ii)]. It follows easily that any element $y \in G W(k)$ may be written as $\operatorname{tr}(A)-\operatorname{tr}(B)$ for $A / k, B / k$ finite étale algebras. Then for $x \in G W^{\times}(k)$ we put $x^{y}:=N_{A / k}\left(\left.x\right|_{A}\right) / N_{B / k}\left(\left.x\right|_{B}\right)$. This division is well-defined because the norm preserves units.

Proposition 22. (i) The element $x^{y} \in G W^{\times}(k)$ is well-defined, independent of the choice of representation $y=\operatorname{tr}(A)-\operatorname{tr}(B)$.

(ii) The pairing

$$
G W(k) \times G W^{\times}(k) \rightarrow G W^{\times}(k),(y, x) \mapsto x^{y}
$$

turns $G W^{\times}(k)$ into a $G W(k)$-module.

(iii) Each of the subgroups $F_{n} G W^{\times}(k) \subset G W^{\times}(k)$ is a $G W(k)$-submodule.

(iv) The $G W(k)$-module $G W^{\times}(k)$ satisfies the projection formulas: For $A / k$ finite étale, we have

$$
N_{A / k}\left(x_{1}^{\left.y_{2}\right|_{A}}\right)=N_{A / k}\left(x_{1}\right)^{y_{2}} \quad \text { for } x_{1} \in G W^{\times}(A), y_{2} \in G W(k)
$$

and

$$
N_{A / k}\left(\left(\left.x_{2}\right|_{A}\right)^{y_{1}}\right)=x_{2}^{t r_{A / k}\left(y_{1}\right)} \quad \text { for } y_{1} \in G W(A), x_{2} \in G W^{\times}(k) .
$$

Proof. Let us note first that if $A, B$ are finite étale $k$-algebras and $(x, y) \in G W(A \times B)=G W(A) \times$ $G W(B)$, then $N_{A \times B / k}(x, y)=N_{A / k}(x) N_{B / k}(y)$. This follows from transitivity of the norm and Proposition 6 by factoring $k \rightarrow A \times B$ as $k \rightarrow k \times k \rightarrow A \times B$ where the first map is the diagonal.

(i) If $\operatorname{tr}(A)-\operatorname{tr}(B)=\operatorname{tr}\left(A^{\prime}\right)-\operatorname{tr}\left(B^{\prime}\right)$ then $\operatorname{tr}\left(A \times B^{\prime}\right)=\operatorname{tr}(A)+\operatorname{tr}\left(B^{\prime}\right)=\operatorname{tr}\left(A^{\prime}\right)+\operatorname{tr}(B)=\operatorname{tr}\left(A^{\prime} \times B\right)$ and consequently

$$
N_{A / k}\left(\left.x\right|_{A}\right) N_{B^{\prime} / k}\left(\left.x\right|_{B^{\prime}}\right)=N_{A \times B^{\prime} / k}\left(\left.x\right|_{A \times B^{\prime}}\right)=N_{A^{\prime} \times B / k}\left(\left.x\right|_{A^{\prime} \times B}\right)=N_{A^{\prime} / k}\left(\left.x\right|_{A^{\prime}}\right) N_{B / k}\left(\left.x\right|_{B}\right),
$$

where for the middle equality we have used Lemma 20, and for the outer equalities we use the first paragraph of this proof. The claim follows upon division by $N_{B^{\prime} / k}\left(\left.x\right|_{B^{\prime}}\right) N_{B / k}\left(\left.x\right|_{B}\right)$.

(ii) Write $y=\operatorname{tr}(A)-\operatorname{tr}(B)$. We have

$$
\left(x_{1} x_{2}\right)^{y}=N_{A / k}\left(x_{1} x_{2}\right) / N_{B / k}\left(x_{1} x_{2}\right)=N_{A / k}\left(x_{1}\right) / N_{B / k}\left(x_{1}\right) \cdot N_{A / k}\left(x_{2}\right) / N_{B / k}\left(x_{2}\right)=x_{1}^{y} x_{2}^{y},
$$

by multiplicativity of the norm. In order to prove that $x^{y_{1}+y_{2}}=x^{y_{1}} x^{y_{2}}$ it is enough to show that if $A / k, B / k$ are finite étale then $N_{A / k}\left(\left.x\right|_{A}\right) N_{B / k}\left(\left.x\right|_{B}\right)=N_{A \times B / k}\left(\left.x\right|_{A \times B}\right)$, since $\operatorname{tr}(A \times B)=\operatorname{tr}(A)+\operatorname{tr}(B)$. This follows from the first paragraph of this proof. Since $\operatorname{tr}(k)=1$ we find $x^{1}=N_{k / k}(x)=x$. Also $x^{0}=1$ for all $x$, since $0=\operatorname{tr}(\emptyset)$ and the norm is multiplicative. It remains to show that $x^{y z}=\left(x^{y}\right)^{z}$. Write $y=\operatorname{tr}(A)-\operatorname{tr}(B)$ and $z=\operatorname{tr}\left(A^{\prime}\right)-\operatorname{tr}\left(B^{\prime}\right)$. Then

$$
\begin{aligned}
& x^{y z}=x^{(\operatorname{tr}(A)-\operatorname{tr}(B))\left(\operatorname{tr}\left(A^{\prime}\right)-\operatorname{tr}\left(B^{\prime}\right)\right)}=x^{\operatorname{tr}(A) \operatorname{tr}\left(A^{\prime}\right)+\operatorname{tr}(B) \operatorname{tr}\left(B^{\prime}\right)-\operatorname{tr}(A) \operatorname{tr}\left(B^{\prime}\right)-\operatorname{tr}\left(A^{\prime}\right) \operatorname{tr}(B)} \\
& =\frac{x^{\operatorname{tr}(A) \operatorname{tr}\left(A^{\prime}\right)} x^{\operatorname{tr}(B) \operatorname{tr}\left(B^{\prime}\right)}}{x^{\operatorname{tr}(A) \operatorname{tr}\left(B^{\prime}\right)} x^{\operatorname{tr}\left(A^{\prime}\right) \operatorname{tr}(B)}}
\end{aligned}
$$

whereas

$$
\begin{gathered}
\left(x^{y}\right)^{z}=\left(x^{\operatorname{tr}(A)-\operatorname{tr}(B)}\right)^{\operatorname{tr}\left(A^{\prime}\right)-\operatorname{tr}\left(B^{\prime}\right)}=\left(x^{\operatorname{tr}(A)} / x^{\operatorname{tr}(B)}\right)^{\operatorname{tr}\left(A^{\prime}\right)-\operatorname{tr}\left(B^{\prime}\right)} \\
=\frac{\left(x^{\operatorname{tr}(A)}\right)^{\operatorname{tr}\left(A^{\prime}\right)}\left(x^{\operatorname{tr}(B)}\right)^{\operatorname{tr}\left(B^{\prime}\right)}}{\left(x^{\operatorname{tr}(B)}\right)^{\operatorname{tr}\left(A^{\prime}\right)}\left(x^{\operatorname{tr}(A)}\right)^{\operatorname{tr}\left(B^{\prime}\right)}},
\end{gathered}
$$

by what we have already established. It is thus enough to show that if $A / k, B / k$ are finite étale then $x^{\operatorname{tr}(A) \operatorname{tr}(B)}=\left(x^{\operatorname{tr}(A)}\right)^{\operatorname{tr}(B)}$. Note that $\operatorname{tr}\left(A \otimes_{k} B\right)=\operatorname{tr}(A) \operatorname{tr}(B)$; this follows for example from the base change formula (for additive transfers). Let $t \in G W(A)$. Then by the base change formula (see Definition 8 part (4) and Corollary 13) we get $\left.\left(N_{A / k}(t)\right)\right|_{B}=N_{A \otimes_{k} B / B}\left(\left.t\right|_{A \otimes_{k} B}\right)$. Substituting $t=\left.x\right|_{A}$, applying $N_{B / k}$ and using transitivity of the norm we get $N_{B / k}\left(\left.\left(N_{A / k}\left(\left.x\right|_{A}\right)\right)\right|_{B}\right)=N_{A \otimes_{k} B / k}\left(\left.x\right|_{A \otimes_{k} B}\right)$. The left hand side is by definition $\left(x^{\operatorname{tr}(A)}\right)^{\operatorname{tr}(B)}$, whereas the right hand side is by definition $x^{\operatorname{tr}(A \otimes B)}$, which is $x^{\operatorname{tr}(A) \operatorname{tr}(B)}$. Thus we have established the desired result.

(iii) It suffices to show that for $A / k$ finite étale we have $N_{A / k}\left(\left.F_{n} G W^{\times}(k)\right|_{A}\right) \subset F_{n} G W^{\times}(k)$. This is immediate from Proposition 17 and the fact that restriction preserves the filtration $F_{n}$.

(iv) We first establish the second claim. By (i) and (ii), both sides are linear in $y_{1}$. We may thus assume that $y_{1}=\operatorname{tr}_{B / A}(1)$, for some $B / A$ finite étale. Then $N_{A / k}\left(\left(\left.x_{2}\right|_{A}\right)^{y_{1}}\right)=N_{A / k}\left(N_{B / A}\left(\left.x_{2}\right|_{B}\right)\right)$ which 
equals $N_{B / k}\left(\left.x_{2}\right|_{B}\right)$ by transitivity, which is the same as $x_{2}^{t r_{k}(B)}$ by definition. The second claim follows since $\operatorname{tr}_{k}(B)=\operatorname{tr}_{A / k}\left(\operatorname{tr}_{B / A}(1)\right)$, by transitivity of transfer. For the first claim, we may assume that $y_{2}=\operatorname{tr}(C)$, with $C / k$ finite étale. Then $\left.y_{2}\right|_{A}=\operatorname{tr}_{C \otimes_{k} A / A}(1)$, by the base change formula (for additive transfers). Thus $N_{A / k}\left(x_{1}^{\left.y_{2}\right|_{A}}\right)=N_{A / k}\left(N_{C \otimes_{k} A / A}\left(\left.x_{1}\right|_{C \otimes_{k} A}\right)\right)$. By transitivity of the norm, this is the same as $N_{C \otimes_{k} A / k}\left(\left.x_{1}\right|_{C \otimes_{k} A}\right)=N_{C / k} N_{C \otimes_{k} A / C}\left(\left.x_{1}\right|_{C \otimes_{k} A}\right)$. By using the base change formula again, we deduce that $N_{C \otimes_{k} A / C}\left(\left.x_{1}\right|_{C \otimes_{k} A}\right)=\left.N_{A / k}\left(x_{1}\right)\right|_{C}$. Putting everything together, we find that

$$
N_{A / k}\left(x_{1}^{\left.y_{2}\right|_{A}}\right)=\left.N_{C / k} N_{A / k}\left(x_{1}\right)\right|_{C}=\left(N_{A / k}\left(x_{1}\right)\right)^{y_{2}} .
$$

This was to be shown.

Proposition 23. (i) Suppose that $1+x \in F_{n} G W^{\times}(k)$, and $y \in G W(k)$. Then $(1+x)^{y} \equiv 1+x y$ $\left(\bmod I^{2 n}(k)\right)$.

(ii) If $A / k$ is of degree 2, then $(-1)^{\operatorname{tr}(A)}=\operatorname{tr}(A)-1$.

(iii) If $y \in I(k)$ then $(-1)^{y} \equiv 1+y\left(\bmod I^{2}\right)$.

(iv) For any $n, m \geq 0$ we have $\left(F_{n} G W^{\times}(k)\right)^{I^{m}(k)} \subset F_{n+m} G W^{\times}(k)$.

Proof. (i) Recall the map $\alpha_{n}: F_{n} G W^{\times} \rightarrow I^{n} / I^{2 n}, x \mapsto x-1$ from equation (11). What we are trying to show is equivalent to $\alpha_{n}\left((1+x)^{y}\right)=\alpha_{n}(1+x) y$, i.e. that $\alpha_{n}$ is a $G W(k)$-module homomorphism. This may be checked on an additive set of generators of $G W(k)$, since we already know that $\alpha_{n}$ is a homomorphism of abelian groups. Thus we may assume that $y=\operatorname{tr}(A)$ for $A / k$ finite étale. In this case the claim is immediate from Proposition 17 part (iii) and the projection formula for additive transfers.

(ii) Using Lemma 16, we compute

$$
0=N_{A / k}(0)=N_{A / k}(1+(-1))=1+N_{A / k}(-1)-\operatorname{tr}_{A / k}(1) .
$$

The result follows by rearranging.

(iii) Let $y \in G W(k)$. It follows from Remark [19] that we have $\operatorname{dim}\left((-1)^{y}\right)=(-1)^{\operatorname{dim}(y)}$. Hence if $y \in I(k)$ then $\operatorname{dim}\left((-1)^{y}\right)=1$ and so $(-1)^{y} \in F_{1} G W^{\times}(k)$. We now have the two maps $f, g: I(k) \rightarrow$ $I(k) / I(k)^{2}$ given by $f(y)=[y]$ and $g(y)=\alpha_{1}\left((-1)^{y}\right)$, and we wish to show that they are equal. Both are group homomorphisms (the second on by Proposition 22 (ii)), so we need only check this on generators. Generators of $I(k)$ are given by $\operatorname{tr}(A)-2$ for $A / k$ degree 2 . Indeed we know that any element of $G W(k)$ can be written as

$$
x=\sum_{i=0}^{n_{1}} \operatorname{tr}\left(A_{i}\right)-\sum_{j=0}^{n_{2}} \operatorname{tr}\left(B_{j}\right)+c
$$

for degree 2 extensions $A_{i}, B_{j} / k$ and $c \in\{0,1\}$. Then $\operatorname{dim}(x)=2\left(n_{1}-n_{2}\right)+c=0$ if and only if $c=0$ and $n_{1}=n_{2}=: n$. In this case we have

$$
x=\sum_{i=0}^{n}\left[\left(\operatorname{tr}\left(A_{i}\right)-2\right)-\left(\operatorname{tr}\left(B_{i}\right)-2\right)\right] .
$$

Now if $A / k$ is degree 2 , then by (ii) we have $(-1)^{\operatorname{tr}(A)-2}=(-1)^{\operatorname{tr}(A)}=\operatorname{tr}(A)-1=1+(\operatorname{tr}(A)-2)$. The claim follows.

(iv) The case $m=0$ is Proposition 22 part (iii). The case $m>1$ follows from $m=1$ and induction. So suppose $m=1$; i.e. we need to show that $\left(F_{n} G W(k)^{\times}\right)^{I(k)} \subset F_{n+1} G W^{\times}(k)$. For $n \geq 1$ this is immediate from (i). We now deal with $n=0$. Thus let $x \in G W^{\times}(k)$. Then $\operatorname{dim}(x) \in\{ \pm 1\}$. Note that $x \in F_{1} G W^{\times}(k)$ if and only if $\operatorname{dim}(x)=1$. Let $y \in I(k)$. Then $\operatorname{dim}(y)=0$, whence $\operatorname{dim}\left(x^{y}\right)=\operatorname{dim}(x)^{y}=1$ as a consequence of Remark 19. It follows that $x^{y} \in F_{1} G W^{\times}(k)$. This concludes the proof.

Remark 24. It follows from part (iv) of Proposition 23 that $(-1)^{I^{2}(k)} \subset F_{2} G W^{\times}(k)$. If $\sqrt{2} \in k$ then one may show that actually $(-1)^{I^{2}(k)}=1$. This follows from Lemma 43 and Theorem 40 In fact the proof of Lemma 43 shows that $(-1)^{(\langle a\rangle-1)(\langle b\rangle-1)}=1+(\langle 2\rangle-1)(\langle a\rangle-1)(\langle b\rangle-1)$, from which we also see that $(-1)^{I^{2}(k)} \neq 1$ in general.

We can use the results of this section to give a presentation of $G W^{\times}(k)$. 
Proposition 25. The $G W(k)$-module $G W^{\times}(k)$ is generated by $F_{2} G W^{\times}(k)$ and -1 . Moreover the following sequence is exact:

$$
I^{2}(k) \stackrel{p \oplus q}{\longrightarrow} G W(k) / 2 \oplus F_{2} G W^{\times}(k) \stackrel{r / s}{\longrightarrow} G W^{\times}(k) \rightarrow 1
$$

Here $p: I^{2}(k) \hookrightarrow G W(k) \rightarrow G W(k) / 2$ is the canonical map, $q(y)=(-1)^{y}, r(x)=(-1)^{x}, s:$ $F_{2} G W^{\times}(k) \hookrightarrow G W^{\times}(k)$ is the canonical inclusion, and $(r / s)(x):=r(x) / s(x)$

Proof. Note that $(-1)^{2}=1$ so $r$ is well-defined. Moreover if $y \in I^{2}(k)$ then $(-1)^{y} \in F_{2} G W^{\times}(k)$ by Proposition 23 part (iv) (or (iii)), so $q$ is well-defined.

To show the claim about generation, or equivalently surjectivity of $r / s$, it suffices to show that any $x \in G W^{\times}(k)$ can be written as $(-1)^{y} z$, with $y \in G W(k)$ and $z \in F_{2} G W^{\times}(k)$. Certainly $\operatorname{dim}(x)= \pm 1$, so $x=(-1)^{n} z_{1}$ for some $n \in \mathbb{Z}$ and $z_{1} \in F_{1} G W^{\times}(k)$. Now $\alpha_{1}\left(z_{1}\right) \in I(k) / I^{2}(k)$. Pick $t \in I(k)$ with $[t]=-\alpha_{1}\left(z_{1}\right)$. Then $\alpha_{1}\left[(-1)^{t} z_{1}\right]=0$ by Proposition 23 part (iii), and so $(-1)^{t} z_{1} \in F_{2} G W^{\times}(k)$. Consequently $x=(-1)^{n-t}\left((-1)^{t} z_{1}\right)$ is of the required form.

It remains to verify exactness in the middle. It is clear that the composite of the two maps is 0 . Now let $x \in G W(k) / 2$. It suffices to show that $(-1)^{x} \in F_{2} G W^{\times}(k)$ only if $x$ is in the image of $p$. Hence suppose that $(-1)^{x} \in F_{2} G W^{\times}(k)$. Then $1=\operatorname{dim}\left((-1)^{x}\right)=(-1)^{\operatorname{dim}(x)}$ and so $\operatorname{dim}(x)$ is even, whence we may assume that $x \in I$. Now $0=\alpha_{1}\left((-1)^{x}\right) \equiv x\left(\bmod I^{2}\right)$ by Proposition 23 part (iii) again, and so $x \in I^{2}$. This concludes the proof.

Remark 26. For a more optimal form of this proposition, see Theorem 44

\section{The sheaf $\underline{G W^{\times}}$and the homotopy module $F_{*}$}

Throughout this section, unless stated otherwise, the field $k$ is assumed perfect. As always, we assume that $\operatorname{char}(k) \neq 2$. We will use various results about strictly homotopy invariant sheaves and homotopy modules. Confer Appendix $\mathrm{A}$ for some recollections on this material.

Recall that the construction $X \mapsto G W(X)$ defines a presheaf on the category of schemes. In the previous section, we have studied its restriction to the subcategory Fét/k of finite étale $k$-schemes. From now on, we will study it on all of $S m(k)$, the category of smooth (separated) $k$-schemes. The associated sheaf (in the Nisnevich or Zariski topology) is denoted $\underline{G W}$, is called the sheaf of unramified Grothendieck-Witt groups, and is strictly homotopy invariant. Recall that a sheaf $F$ on $S m(k)$ is called strictly homotopy invariant if the canonical map $H_{N i s}^{p}(X, F) \rightarrow H_{N i s}^{p}\left(X \times \mathbb{A}^{1}, F\right)$ is an isomorphism for all $X \in S m(k)$ and all $p \geq 0$. The sheaf $\underline{G W}$ coincides with the sheaf constructed by Morel 20, Section 3.2] [21, Theorem A]. Note also that $\underline{\left.G W\right|_{F e ́ t} / k}=\left.G W\right|_{F e ́ t / k}$, since all finite étale $k$-schemes are (finite) disjoint unions of Nisnevich local schemes. Since $\underline{G W}$ is a sheaf of rings, it has a subsheaf of units which we denote $\underline{G W^{\times}}$. This is the sheaf associated with $S m(k) \ni X \mapsto G W(X)^{\times}$. Our first task is to prove that $\underline{G W^{\times}}$is also strictly homotopy invariant.

In order to do this, we recall that there are the sheaves of ideals $\underline{I}^{n} \subset \underline{G W}$. We define a filtration of $\underline{G W^{\times}}$via $F_{n} \underline{G W}^{\times}(X)=\left(1+\underline{I}^{n}(X)\right) \cap \underline{G W}^{\times}(X)$. As before we get homomorphisms $\alpha_{n}: F_{n} \underline{G W}^{\times} \rightarrow$ $\underline{I}^{n} / \underline{I}^{2 n}$, where on the right hand side we mean the quotient taken in the category of Nisnevich sheaves.

If $F$ is any (pre)sheaf on $S m(k)$ we write $F_{t o r}$ for the (pre)sheaf $F_{t o r}(X)=F(X)_{t o r}$, where for an abelian group $A$ we write $A_{\text {tor }}$ for the subgroup of torsion elements. It is strictly homotopy invariant if $F$ is. This follows from the fact that the category of strictly homotopy invariant sheaves is abelian and closed under filtered colimits.

Lemma 27. If $n \geq 2$ then $F_{n} \underline{G W^{\times}}=1+\underline{I}_{t o r}^{n}$, where the identification holds as sub-presheaves of $\underline{G W}$.

Proof. Let $1+x \in 1+\underline{I}^{n}(X) \subset \underline{G W}(X)$. We need to show that $1+x \in \underline{G W^{\times}}(X)$ if and only if $x$ is torsion. I claim that $x$ is torsion if and only if it is nilpotent. Indeed since $\underline{G W}$ is strictly homotopy invariant it is unramified [19, Lemma 6.4.4], and thus it suffices to prove the claim for $G W(L)$ with $L$ a field, where it follows from [15, Theorems III.3.6 and III.3.8]. We thus need to show that $1+x \in \underline{G W}(X)$ is invertible if and only if $x$ is nilpotent. Certainly if $x$ is nilpotent then $1+x$ is invertible. Conversely, if $1+x$ is invertible then so is its image in $G W(L)$ for any field $L$, and then by unramifiedness of $\underline{G W}$ again it suffices to prove: if $1+x \in G W(L)$ is invertible with $x \in I^{n}(L)$ and $n \geq 2$, then $x$ is nilpotent (or equivalently, torsion). Let $\sigma: G W(L) \rightarrow \mathbb{Z}$ be a signature map. By [15, Theorems III.3.6 and III.3.8] again it suffices to show that $\sigma(x)=0$. But $\sigma(I) \subset 2 \mathbb{Z}$ and hence $\sigma(x) \in 2^{n} \mathbb{Z}$, whereas also $\sigma(1+x)=1+\sigma(x) \in \mathbb{Z}^{\times}=\{ \pm 1\}$. As $n \geq 2$ this implies that $\sigma(x)=0$, as was to be shown. 
Lemma 28. We have

$$
\underline{G W^{\times}} / F_{1} \underline{G W^{\times}} \cong \mathbb{Z} / 2 \cong \underline{k}_{0}^{M},
$$

induced by $\operatorname{dim}: \underline{G W^{\times}} \rightarrow \mathbb{Z}$ (here by $\mathbb{Z}$ and $\mathbb{Z} / 2$ we also denote the associated constant sheaves),

$$
F_{1} \underline{G W}^{\times} / F_{2} \underline{G W} \times \underline{G}_{m} / 2 \cong \underline{k}_{1}^{M} \cong \underline{I} / \underline{I}^{2},
$$

induced by $\alpha_{1}$, and for $n \geq 2$ we have

$$
F_{n} \underline{G W}^{\times} / F_{n+1} \underline{G W}^{\times} \cong \underline{I}_{t o r}^{n} / \underline{I}_{t o r}^{n+1} \hookrightarrow \underline{I}^{n} / \underline{I}^{n+1},
$$

induced by $\alpha_{n}$. In particular all of the subquotients of the filtration are strictly homotopy invariant.

Proof. For $n=0$ the map $\underline{G W^{\times}} / F_{1} \underline{G W^{\times}} \rightarrow(\underline{G W} / \underline{I})^{\times}=\mathbb{Z} / 2$ is an isomorphism: it is surjective since it has a section and it is injective because $F_{1} \underline{G W^{\times}}=(1+\underline{I}) \cap \underline{G W}^{\times}$by definition.

For $n \geq 1$ the map $\alpha_{n}$ satisfies $\alpha_{n}^{-1}\left(\underline{I}^{n+1} / \underline{I}^{2} n\right)=F_{n+1} \underline{G W}^{\times}$and hence induces an injection $\beta_{n}$ : $F_{n} \underline{G W}^{\times} / \bar{F}_{n+1} \underline{G W}^{\times} \hookrightarrow \underline{I}^{n} / \underline{I}^{n+1}$.

There is a homomorphism $\mathbb{G}_{m} / 2 \rightarrow \underline{G W^{\times}}, a \mapsto\langle a\rangle$ splitting $\beta_{1}$, so $\beta_{1}$ is an isomorphism. For $n \geq 2$ by Lemma 27 we have $F_{n} \underline{G W}^{\times}=1+\underline{I}_{t o r}^{n}$ and so the image of $\beta_{n}$ is $\underline{I}_{t o r}^{n} / \underline{I}_{t o r}^{n+1} \subset \underline{I}^{n} / \underline{I}^{n+1}$, as claimed.

For the last claim, since each $\underline{I}^{n}$ is strictly homotopy invariant [20, Example 3.34] so is $\underline{I}_{\text {tor }}^{n}$, and hence so is the quotient $\underline{I}_{\text {tor }}^{n} / \underline{I}_{\text {tor }}^{n+\overline{1}}$. Here we have used again that the category of strictly homotopy invariant sheaves is abelian and closed under filtered colimits.

We will repeatedly use the following result, essentially due to Elman and Lum.

Lemma 29 (Elman and Lum [6]). Let $k$ be a field such that $\operatorname{char}(k) \neq 2$ and $v_{c} d_{2}(k)<n$. Then $I_{\text {tor }}^{n}(k)=0$ and in particular $2^{n} I_{\text {tor }}^{r}(k)=0$ for all $r>0$ (and also $\left.2^{n} W_{\text {tor }}(k)=0\right)$.

Proof. Applying (vi) of the last theorem of [6] to $K=k(T), F=k$ gives the first statement. The remainder follows from $2 \in I(k) \subset W(k)$.

Theorem 30. Let $k$ be any field with char $(k) \neq 2$. Then the sheaf $\underline{G W^{\times}}$(on $\left.\operatorname{Sm}(k)\right)$ is strictly homotopy invariant. The same is true for $F_{r} \underline{G W^{\times}}$for any $r$.

Proof. Suppose first that $v c d_{2}(k)<n$ and $k$ is perfect. Then for any field $L / k$ of transcendence degree at most $m$ over $k$ we have $v_{c d}(k)<n+m$ [25. Theorem 28 of Chapter 4] and so $I_{\text {tor }}^{n+m}(L)=0$ by Lemma 29.

Let $X \in S m(k)$ be of dimension at most $m$. It follows from the first paragraph and unramifiedness that $\left.F_{n+m} \underline{G W^{\times}}\right|_{X_{N i s}}=1$. Hence on $X$ (and on $X \times \mathbb{A}^{1}$ ) the sheaf $\underline{G W}{ }^{\times}$is a finite extension of strictly homotopy invariant sheaves, by Lemma 28 , and consequently is strictly homotopy invariant.

The same argument works for $F_{r} \underline{G W^{\times}}$for $r \neq 0$.

For the general case in which $v c d_{2}(k)$ might be infinite and $k$ need not be perfect we use a continuity argument. Let $k_{0} \subset k$ be the prime subfield and write $p: \operatorname{Spec}(k) \rightarrow \operatorname{Spec}\left(k_{0}\right)$ for the canonical morphism. Then $k_{0}$ is perfect and $v c d_{2}(k)<\infty$. The morphism $p$ is essentially smooth by [10, Lemma A.2]. Hence by Lemma 51 we find that $\left.\underline{G W}\right|_{S m(k)}=p^{*}\left(\left.\underline{G W}\right|_{S m\left(k_{0}\right)}\right)$ and thus also $\left.F_{r} \underline{G W^{\times}}\right|_{S m(k)}=p^{*}\left(\left.F_{r} \underline{G W^{\times}}\right|_{S m\left(k_{0}\right)}\right)$. Since $p^{*}$ preserves strictly homotopy invariant sheaves [10, Lemma A.4], this concludes the proof.

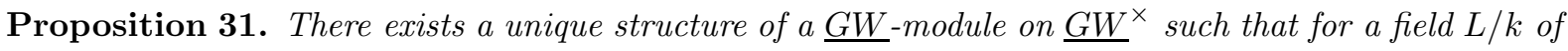
finite transcendence degree, the induced $G W(L)$-module structure on $G W^{\times}(L)$ is the one from Section 4.

Proof. Uniqueness follows from unramifiedness of $\underline{G W}$. For existence, let $x \in \underline{G W}(X)$ and $y \in \underline{G W^{\times}}(X)$. Write $a: X^{(0)} \rightarrow X$ for the inclusion of the generic points. We need to show that $\left(a^{*} y\right)^{a^{*} x} \in \underline{G W^{\times}}(X) \subset$ $\underline{G W^{\times}}\left(X^{(0)}\right)$ is an unramified element. Indeed, recall (possibly from Appendix $\mathrm{A}$ ) that if $F$ is an unramified sheaf and $X$ is connected (hence irreducible), we have

$$
F(X)=\bigcap_{x \in X^{(1)}} F\left(X_{x}\right) \subset F(k(X)) .
$$

In other words we need to prove that $\left(a^{*} y\right)^{a^{*} x} \in \underline{G W^{\times}}(X)$ whenever $X$ is the spectrum of a dvr (or more generally local ring). In this case $\underline{G W}(X)=G W(X)$ is generated by the one-dimensional diagonal forms $\langle a\rangle$ with $a \in \mathcal{O}^{\times}(X)$ [15, Corollary I.3.4] and consequently the traces of étale $X$-schemes generate $G W(X)$, by the same argument as before Proposition 22. Let $x=\operatorname{tr}\left(Y_{1}\right)-\operatorname{tr}\left(Y_{2}\right)$. Then $\left(a^{*} y\right)^{a^{*} x}=a^{*}\left(N_{Y_{1} / X}\left(\left.y\right|_{Y_{1}}\right) / N_{Y_{2} / X}\left(\left.y\right|_{Y_{2}}\right)\right)$. Since $N_{Y_{1} / X}\left(\left.y\right|_{Y_{1}}\right), N_{Y_{2} / X}\left(\left.y\right|_{Y_{2}}\right) \in G W(X)^{\times}$, this concludes the proof. 
By unramifiedness, the results from Section 4 over fields immediately extend to all of $\underline{G W}$ :

Corollary 32. (i) Each of the subsheaves $F_{n} \underline{G W^{\times}} \subset \underline{G W^{\times}}$is a sub-GW-module.

(ii) For any $n, m \geq 0$ we have $\left(F_{n} \underline{G W^{\times}}\right)^{\underline{I}^{m}} \subset F_{n+m} \underline{G W^{\times}}$.

Proof. For (i), if $x \in \underline{G W}(X)$ and $y \in F_{n} \underline{G W^{\times}}(X)$ then we wish to show that $y^{x} \in F_{n} \underline{G W} \times(X)$, where $y^{x} \in G W^{\times}(X)$ is defined using the module structure established in Proposition 31. But by definition $F_{n} \underline{G W}^{\times}(X)=1+\underline{I}^{n}(X) \cap \underline{G W}^{\times}(X)$, and $\underline{I}^{n}(X)=\underline{I}^{n}\left(X^{(0)}\right) \cap \underline{G W}(X)$ as a consequence of Lemma 48. It follows that $F_{n} \underline{G W^{\times}}(X)=\underline{G W^{\times}}(X) \cap F_{n} G W^{\times}\left(X^{(0)}\right)$. We are thus reduced to showing that $y^{x} \in F_{n} G W^{\times}\left(X^{(0)}\right)$. This is Proposition 22 part (iii).

The argument for (ii) is the same, using Proposition 23 part (iv).

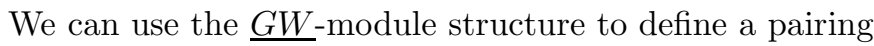

$$
\beta: \mathbb{Z}\left[\mathbb{G}_{m}\right] \otimes \underline{G W}^{\times} \rightarrow \underline{G W^{\times}},(u, x) \mapsto x^{\langle u\rangle-1} .
$$

Since $\langle u\rangle-1 \in \underline{I}$, by Corollary $\left[32\right.$ we know that $\beta\left(\mathbb{Z}\left[\mathbb{G}_{m}\right] \otimes F_{n} \underline{G W^{\times}}\right) \subset F_{n+1} \underline{G W^{\times}}$. We write $\beta_{n}$ : $\mathbb{Z}\left[\mathbb{G}_{m}\right] \otimes F_{n} \underline{G W}^{\times} \rightarrow F_{n+1} \underline{G W^{\times}}$for this restricted pairing. By adjunction, $\beta_{n}$ induces a homomorphism $\beta_{n}^{\dagger}: F_{n} \underline{G W^{\times}} \rightarrow\left(F_{n+1} \underline{G W^{\times}}\right)_{-1}$. Here for a presheaf $F$ we denote by $F_{-1}=\underline{\operatorname{Hom}}\left(\mathbb{Z}\left[\mathbb{G}_{m}\right], F\right)$ its contraction; see Appendix $\mathrm{A}$ for more on this construction.

Proposition 33. Let $k$ be a field of characteristic different from 2. For $n \geq 2$ the homomorphism $\beta_{n}^{\dagger}: F_{n} \underline{G W^{\times}} \rightarrow\left(F_{n+1} \underline{G W^{\times}}\right)_{-1}$ is an isomorphism.

Proof. Throughout we will assume $n \geq 2$. We first assume that $k$ is perfect and $v c d_{2}(k)<\infty$; these assumptions are removed at the end by a continuity argument. The commutative square

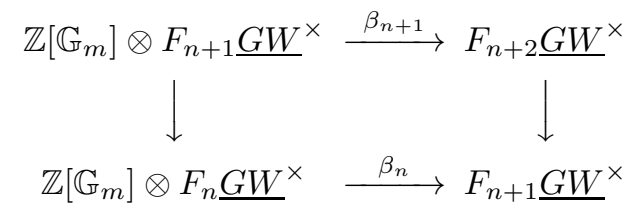

in which the vertical maps are the canonical inclusions, induces by adjunction a commutative square

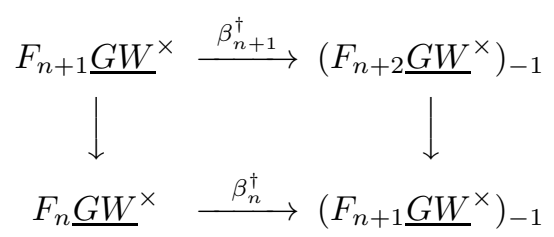

Since contraction is an exact operation [20, Lemma 7.33], by Lemma 28] we get a diagram of short exact sequences

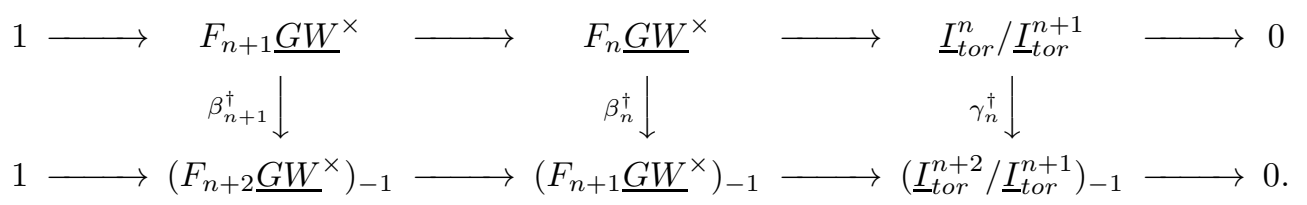

Here $\gamma_{n}^{\dagger}$ is defined so as to make the diagram commute.

I claim that $\gamma_{n}^{\dagger}$ is an isomorphism. To see this, let $\delta_{n}: \mathbb{Z}\left[\mathbb{G}_{m}\right] \otimes \underline{I}^{n} \rightarrow \underline{I}^{n+1}$ be the homomorphism $(u, x) \mapsto(\langle u\rangle-1) x$. Then $\delta_{n}^{\dagger}: \underline{I}^{n} \rightarrow\left(\underline{I}^{n+1}\right)_{-1}$ is an isomorphism. This is just because $\underline{I}^{*}$ is a homotopy module where the element $[u] \in \underline{K}_{1}^{M W}$ acts via $\langle u\rangle-1 \in \underline{I}^{1}$. It follows that the restriction $\delta_{n}^{\dagger}: \underline{I}_{t o r}^{n} \rightarrow\left(\underline{I}_{t o r}^{n+1}\right)_{-1}$ is also an isomorphism. Note that $\left(F_{t o r}\right)_{-1}=\left(F_{-1}\right)_{t o r}$. Now consider the commutative diagram of exact sequences

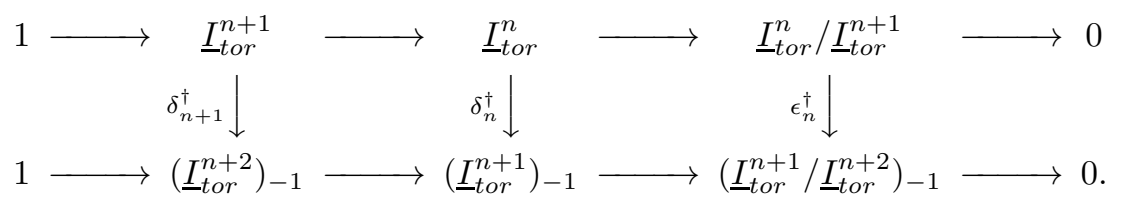


We find that $\epsilon_{n}^{\dagger}$ is an isomorphism, by the 5-lemma. But also $\epsilon_{n}^{\dagger}=\gamma_{n}^{\dagger}$. For this it suffices to show that $\epsilon_{n}=\gamma_{n}$. Since the target is strictly homotopy invariant, hence unramified, it suffices to show that $\epsilon_{n}$ and $\gamma_{n}$ induce the same map on sections over fields. Thus let $L$ be a field. By definition, the following diagram commutes:

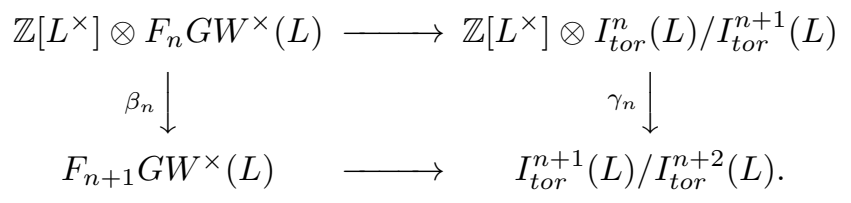

Here the horizontal maps are induced by $x \mapsto x-1$, and $\beta_{n}(a \otimes x)=x^{\langle a\rangle-1}$, for $a \in L^{\times}$and $x \in$ $F_{n} G W^{\times}(L)$. It now follows from Proposition 23 part (i) that for $\bar{x} \in I_{\text {tor }}^{n}(L) / I_{\text {tor }}^{n+1}(L)$ we have $\gamma_{n}(a \otimes \bar{x})=$ $(\langle a\rangle-1) x$. Here we use that $n \geq 2$. By definition, this is the same as $\epsilon_{n}(a \otimes \bar{x})$. Hence $\epsilon_{n}=\gamma_{n}$ and thus $\gamma_{n}^{\dagger}$ is an isomorphism as claimed.

Now in order to show that $\beta_{n}^{\dagger}$ is an isomorphism, it suffices to show that for every field $L$ (of finite transcendence degree over $k$ ) the section $\beta_{n}^{\dagger}(L)$ is an isomorphism (since the kernel and cokernel of $\beta_{n}^{\dagger}$ are strictly homotopy invariant and hence unramified). Recall that we assume for now that $v c d_{2}(k)<\infty$. Then also $v_{c d}(L)<\infty$ and for $n$ sufficiently large we have $F_{n} G W^{\times}(L)=1$, by the Lemmas 27] and 29. In particular for $n$ sufficiently large $\beta_{n}^{\dagger}(L)$ is an isomorphism. We may thus prove that $\beta_{n}^{\dagger}(L)$ is an isomorphism for all $n \geq 2$ by descending induction on $n$. The induction step follows by considering the diagram of exact sequences (2) and using that $\gamma_{n}^{\dagger}$ is an isomorphism, as we established above.

For the general case in which $v_{c d_{2}}(k)$ may be infinite and $k$ may be imperfect, let $p: S p e c(k) \rightarrow$ $\operatorname{Spec}\left(k_{0}\right)$ be an essentially smooth morphism to a perfect field with $v c d_{2}\left(k_{0}\right)<\infty$ (e.g. $k_{0}$ the prime field). It follows from Lemma 50 that $p^{*}$ commutes with contractions, and it follows from Corollary 51 that $p^{*} F_{n} \underline{G W^{\times}}=F_{n} \underline{G W^{\times}}$. Then $\beta_{n}^{\dagger}=p^{*} \beta_{n}^{\dagger}$ is an isomorphism.

We have thus managed to deloop the sheaves $F_{n} G W^{\times}$for $n \geq 2$. Recall (possibly from Appendix A) that a homotopy module consists of a sequence of sheaves $F_{n} \in S h v_{N i s}(\operatorname{Sm}(k))$ together with isomorphisms $F_{n} \cong\left(F_{n+1}\right)_{-1}$, such that each $F_{n}$ is strictly homotopy invariant.

Corollary 34. There is a homotopy module $F_{*}$, determined up to unique isomorphism of homotopy modules, such that for $n \geq 2$ the following hold:

- $F_{n} \cong F_{n} \underline{G W^{\times}}$, and

- the bonding $\operatorname{map} F_{n} \stackrel{\cong}{\longrightarrow}\left(F_{n+1}\right)_{-1}$ equals $\beta_{n}^{\dagger}$.

\section{The logarithm isomorphism}

Throughout this section, the base field $k$ is assumed to be perfect. As always, we assume that it is of characteristic different from 2 .

In this section we shall study in more detail the homotopy module $F_{*}$. Recall from Appendix $\mathrm{A}$ that if $G_{*}$ is any homotopy module, then each $G_{n}$ has the structure of a $\underline{G W}$-module, and also has transfers along finite étale morphisms known as cohomological transfers. Note that the definition of a homotopy module $G_{*}$ only asks for isomorphisms $G_{n} \cong\left(G_{n+1}\right)_{-1}$ and strict homotopy invariance of the $G_{i}$. The transfers and $\underline{G W}$-module structure are implicit in this data. In particular, each of the sheaves $F_{n} \underline{G W^{\times}}$

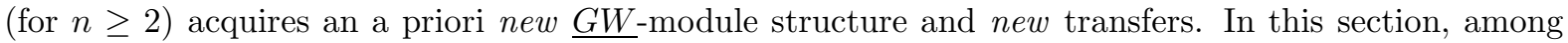
other things, we shall show that the "new" $G W$-module structure on $F_{n}=F_{n} \underline{G W^{\times}}$coincides with that of Section 5, and that the "new" cohomological transfers coincide with Rost's multiplicative transfers. We begin by comparing the module structures. For $X \in S m(k), a \in \underline{G W}(X), x \in F_{*}(X)$ we denote by $a x$ the action of $\underline{G W}(X)$ coming from the fact that $F_{*}$ is a homotopy module, and we denote by $x^{a}$ the action coming from the module structure we constructed in Section 5. What we are trying to prove, then, is that $a x=x^{a}$.

Lemma 35. For $n \geq 2$ the $\underline{G W}$-module structure on $F_{n}=F_{n} \underline{G W}{ }^{\times}$coincides with the module structure defined in Section 5 .

Proof. We first describe the $G W$-module structure on a homotopy module $G_{*}$. Let $L$ be a field of finite transcendence degree over $k$. Let $\mathcal{O} \subset L$ be a geometric dvr with uniformiser $\pi$ and residue field $\kappa$. As explained in Appendix $\mathrm{A}$, there is the boundary map $\partial^{\pi}: G_{n+1}(L) \rightarrow G_{n}(\kappa)$, with kernel $G_{n+1}(\mathcal{O})$. The 
isomorphism $G_{n} \rightarrow\left(G_{n+1}\right)_{-1}$ induces a map $\mathbb{Z}\left[\mathbb{G}_{m}\right] \otimes G_{n} \rightarrow G_{n+1}$ which we denote $(u, x) \mapsto[u] x$. Then by Lemma 47 in the appendix one has

$$
\partial^{\pi}([u \pi] x)=\left\langle s^{*}(u)\right\rangle s^{*}(x)
$$

where $s: \operatorname{Spec}(\kappa) \rightarrow \operatorname{Spec}(\mathcal{O})$ is the inclusion of the closed point, and where on the right hand side multiplication by an element of $G W(\kappa)$ refers to the $G W$-module structure we are describing. If in addition $\mathcal{O}$ is Henselian then $s$ has a section and so $s^{*}$ is surjective, so in this case equation (3) determines the $G W$-module structure on $G_{*}(\kappa)$ uniquely.

Now let $\kappa$ be a field of finite transcendence degree over $k$. It suffices to show that the $G W(\kappa)$-module structure on $F_{n}$ is the one from Section 5. Choose an essentially smooth local curve over $k$ with residue field $\kappa$ (e.g. the localisation of $\mathbb{A}_{\kappa}^{1}$ in the origin). Passing to the Henselization, we obtain a Henselian dvr $\mathcal{O}$ with residue field $\kappa$ and some fraction field $L$, also of finite transcendence degree. Pick a uniformiser $\pi$. We wish to show that for all $\bar{u} \in \kappa^{\times}$and all $\bar{x} \in F_{n}(\kappa)$ we have $\langle\bar{u}\rangle \bar{x}=\bar{x}^{\langle\bar{u}\rangle}$, where on the left hand side we mean the module structure coming from $F_{*}$ being a homotopy module and on the right hand side we mean the module structure constructed in Section [5. By the first paragraph, for this it suffices to show that $\partial^{\pi}([u \pi] x)=s^{*}(x)^{\langle\bar{u}\rangle}$ for all $x \in F_{n}(\mathcal{O})$ and all $u \in \mathcal{O}^{\times}$. We compute

$$
[u \pi] x=x^{\langle u \pi\rangle-1}=x^{\langle u\rangle\langle\pi\rangle-\langle u\rangle+\langle u\rangle-1}=\left(x^{\langle u\rangle}\right)^{\langle\pi\rangle-1} x^{\langle u\rangle-1}=\left([\pi] x^{\langle u\rangle}\right)([u] x) .
$$

Here the first and last equalities are by definition of the homotopy module structure on $F_{*}$. Note that $\partial^{\pi}([\pi] z)=s^{*}(z)$ by equation (3) , and $\partial^{\pi}$ is a homomorphism with kernel $F_{n+1}(\mathcal{O})$. Since $[u] x \in F_{n+1}(\mathcal{O})$ we thus obtain

$$
\partial^{\pi}([u \pi] x)=\partial^{\pi}\left([\pi] x^{\langle u\rangle}\right)=s^{*}\left(x^{\langle u\rangle}\right)=s^{*}(x)^{\langle\bar{u}\rangle} .
$$

This concludes the proof.

What we have done so far has the following interesting consequence.

Lemma 36. Let $n \geq 2, m \geq 0, X \in S m(k)$, and $x \in F_{n} \underline{G W^{\times}}(X)$. Then the element

$$
x^{\left(\left\langle t_{1}\right\rangle-1\right)\left(\left\langle t_{2}\right\rangle-1\right) \ldots\left(\left\langle t_{m}\right\rangle-1\right)} \in F_{n+m} \underline{G W^{\times}}\left(X \times\left(\mathbb{A}^{1} \backslash 0\right)^{m}\right) \subset \underline{G W}\left(X \times\left(\mathbb{A}^{1} \backslash 0\right)^{m}\right)
$$

may be written as

$$
1+\left(\left\langle t_{1}\right\rangle-1\right)\left(\left\langle t_{2}\right\rangle-1\right) \ldots\left(\left\langle t_{m}\right\rangle-1\right) y
$$

for a unique $y \in \underline{I}^{n}(X)$.

This induces a bijection (of sets!) $\log _{(m)}: F_{n} \underline{G W}^{\times}(X) \rightarrow \underline{I}_{t o r}^{n}(X)$.

Proof. Recall that if $G_{*}$ is any homotopy module, then

$$
G_{n+1}\left(X \times\left(\mathbb{A}^{1} \backslash 0\right)\right)=G_{n+1}(X) \oplus\left[t_{1}\right] G_{n}(X),
$$

where $G_{n}(X) \rightarrow\left[t_{1}\right] G_{n}(X)$ is an isomorphism, namely multiplication by $t_{1}$. Moreover in this decomposition, the factor $\left[t_{1}\right] G_{n}(X)$ consists precisely of those $x \in G_{n+1}\left(X \times\left(\mathbb{A}^{1} \backslash 0\right)\right)$ such that $i_{1}^{*}(x)=0$, where $i_{1}: X \rightarrow X \times\left(\mathbb{A}^{1} \backslash 0\right)$ is the inclusion of the point $1 \in \mathbb{A}^{1}$. This is the content of Lemma 46 in the appendix. By induction, the map $\left[t_{1}\right] \ldots\left[t_{m}\right]: G_{n}(X) \rightarrow G_{n}\left(X \times\left(\mathbb{A}^{1} \backslash 0\right)^{m}\right)$ is injective, and its image consists of precisely those $x \in G_{n}\left(X \times\left(\mathbb{A}^{1} \backslash 0\right)^{m}\right)$ such that for each $r \in\{1, \ldots, m\}$ we have $j_{r}^{*}(x)=0$, where $j_{r}: X \times\left(\mathbb{A}^{1} \backslash 0\right)^{m-1} \rightarrow X \times\left(\mathbb{A}^{1} \backslash 0\right)^{m}$ is the inclusion at the point 1 in the $r$-th factor $\mathbb{A}^{1} \backslash 0$.

Applying this to the homotopy module $F_{*}$ we find that

$$
\alpha: F_{n}(X) \rightarrow F_{n+m}\left(X \times\left(\mathbb{A}^{1} \backslash 0\right)^{m}\right), x \mapsto\left[t_{1}\right] \ldots\left[t_{m}\right] x=x^{\left(\left\langle t_{1}\right\rangle-1\right) \ldots\left(\left\langle t_{m}\right\rangle-1\right)}
$$

is an injection with image consisting of those $y \in F_{n+m}\left(X \times\left(\mathbb{A}^{1} \backslash 0\right)^{m}\right) \subset \underline{G W}\left(X \times\left(\mathbb{A}^{1} \backslash 0\right)^{m}\right)$ such that $j_{r}^{*}(y)=1$ for all $r \in\{1,2, \ldots, m\}$. Since $j_{r}^{*}$ is a ring homomorphism, we conclude by Lemma 27 that $\alpha-1$ is a bijection onto the subset of $\underline{I}_{t o r}^{n+m}\left(X \times\left(\mathbb{A}^{1} \backslash 0\right)^{m}\right)$ consisting of those elements such that $j_{r}^{*}=0$ for all $r$. Applying the remark from the first paragraph to the homotopy module $\underline{I}_{\text {tor }}^{*}$ (for which $[u] x=(\langle u\rangle-1) x)$ concludes the proof.

Thus for any $x \in F_{n} \underline{G W}^{\times}(X)$ we obtain a sequence $x=\log _{(0)}(x), \log _{(1)}(x), \log _{(2)}(x), \cdots \in \underline{I}_{\text {tor }}^{n}(X)$. We would like to take the "limit" of this sequence.

For the remainder of this section, we will use the abbreviation $P_{m}:=\prod_{i=1}^{m}\left(\left\langle t_{i}\right\rangle-1\right) \in \underline{G W}\left(\left(\mathbb{A}^{1} \backslash 0\right)^{m}\right)$. 
Remark 37. By Lemma 36 we have $x^{P_{m}}=1+P_{m} \log _{(m)}(x)$. Since multiplication by $P_{m}$ is injective in an appropriate sense (see the previous proof), we may write this as

$$
\log _{(m)}(x)=\left(x^{P_{m}}-1\right) / P_{m} .
$$

We think of $P_{m} \in I^{m}$ as small, and taking the limit we propose corresponds to the formula

$$
\lim _{\epsilon \rightarrow 0+}\left(x^{\epsilon}-1\right) / \epsilon=\log (x)
$$

from classical analysis.

Lemma 38. Let $L^{\prime} / L$ be a degree 2 extension ( $L$ of characteristic not 2$)$.

(i) We have $N_{L^{\prime}(t) / L(t)}(\langle t\rangle-1)=-\operatorname{tr}\left(L^{\prime}\right)(\langle t\rangle-1)$.

(ii) We have $\operatorname{tr}\left(L^{\prime}\right)^{2}=2 \operatorname{tr}\left(L^{\prime}\right)$.

(iii) There exists $y \in G W(L)$ such that $y N_{L^{\prime} / L}(2)=8$.

Proof. (i) By Lemma 16 we get $N(\langle t\rangle-1)=N(\langle t\rangle)-\operatorname{tr}(1)\langle t\rangle+N(-1)$, where we have used that $\operatorname{tr}(-\overline{\langle t\rangle})=-\langle t\rangle \operatorname{tr}(1)$. Note that $N(\langle t\rangle)=\left\langle t^{2}\right\rangle=1$ : this is clear if $A=k \times k$, and for the quadratic case see [29, Lemma 2.6(ii)]. Since $N(-1)=(-1)^{\operatorname{tr}(1)}=\operatorname{tr}(1)-1$ by Proposition 23 part (ii), the result follows from the observation that $\operatorname{tr}_{L^{\prime}(t) / L(t)}(1)=\left.\operatorname{tr}_{L^{\prime} / L}(1)\right|_{L(t)}$, i.e. the base change formula.

(ii) Since $\operatorname{tr}\left(L^{\prime}\right)-1=N(-1)$ we get $\left(\operatorname{tr}\left(L^{\prime}\right)-1\right)^{2}=1$. The result follows.

(iii) We have $N(2)=N(1+1)=2+\operatorname{tr}(1)$ by Lemma 16 again. Thus if we put $\xi=N(2)$ then by (ii) we find $(\xi-2)^{2}=2(\xi-2)$ which implies that $\xi(6-\xi)=8$.

Corollary 39. Let $L^{\prime} / L$ be a degree 2 extension with $\operatorname{char}(L) \neq 2, x \in G W(L)$ and $2^{r} x=0$. Then for $m>3 r$ we have

$$
N_{L^{\prime}\left(t_{1}, \ldots, t_{m}\right) / L\left(t_{1}, \ldots, t_{m}\right)}\left(1+P_{m} x\right)=1+P_{m} \operatorname{tr}_{L^{\prime} / L}(x) .
$$

Proof. Using Lemma [16 we compute that $N\left(1+P_{m} x\right)=1+\operatorname{tr}\left(P_{m} x\right)+N\left(P_{m}\right) N(x)$. Noting that $\operatorname{tr}_{L^{\prime}\left(t_{1}, \ldots, t_{m}\right) / L\left(t_{1}, \ldots, t_{m}\right)}\left(P_{m} x\right)=P_{m} t_{L^{\prime} / L}(x)$ by the base change and projection formulas, we thus need to show that $N\left(P_{m}\right) N(x)=0$. Since $2^{r} x=0$ we get $0=N\left(2^{r} x\right)=N\left(2^{r}\right) N(x)$, and hence by Lemma 38 part (iii) we find that $8^{r} N(x)=0$. I claim that $N\left(P_{m}\right)=(-1)^{m} 2^{m-1} \operatorname{tr}\left(L^{\prime}\right) P_{m}$. Since $m>3 r$ the claim implies that $N\left(P_{m}\right)$ is divisible by $8^{r}$ and hence $N\left(P_{m}\right) N(x)=0$ as needed.

To prove the claim, note that $N\left(P_{m}\right)=\prod_{i=1}^{m} N\left(\left\langle t_{i}\right\rangle-1\right)=\left(-\operatorname{tr}\left(L^{\prime}\right)\right)^{m} P_{m}$, by Lemma 38 part (i), and $\left(-\operatorname{tr}\left(L^{\prime}\right)\right)^{m}=(-1)^{m} 2^{m-1} \operatorname{tr}\left(L^{\prime}\right)$ by Lemma 38 part (ii).

Theorem 40. For $n \geq 2$ and $x \in F_{n} \underline{G W}^{\times}(X)$, the sequence $\log _{(m)}(x) \in \underline{I}_{t o r}^{n}(x)$ is eventually constant. Write $\log (x)$ for this eventual value. This defines an isomorphisms of homotopy modules $\log : F_{*} \rightarrow \underline{I}_{\text {tor }}^{*}$ such that for any $n \geq 2$ and any finite separable extension $L^{\prime} / L$ with $L$ of finite transcendence degree over $k$, the following diagram commutes

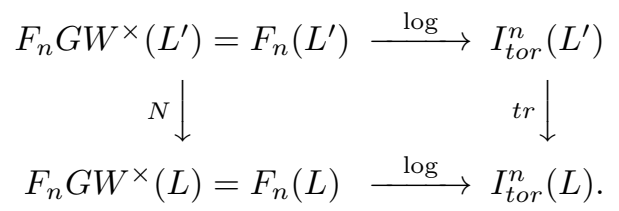

Here on the left hand side $N$ denotes Rost's multiplicative transfer, whereas on the right hand side tr denotes the cohomological transfer present on any homotopy module, see Appendix A. In particular the cohomological transfer on $F_{n}$ coincides with Rost's multiplicative transfer.

Proof. It suffices to show the following: (a) for each $n \geq 2$ there is a well-defined morphism of sheaves of abelian groups $\log : F_{n} \rightarrow \underline{I}_{\text {tor }}^{n}$, (b) the maps log are compatible with the isomorphisms $F_{n} \cong\left(F_{n+1}\right)_{-1}$ and $\underline{I}^{n} \cong\left(\underline{I}^{n+1}\right)_{-1}$. This implies that there is indeed a morphism of homotopy modules as stated. We then need to check for each $n \geq 2$ that (c) the map $\log : F_{n} \rightarrow \underline{I}_{t o r}^{n}$ is an isomorphism, and that it is (d) compatible with the transfers, in the sense of equation (4).

For now we shall assume that $v c d_{2}(k)<\infty$. We will remove this assumption at the end by a continuity argument. Let us also fix $n \geq 2, X \in S m(k)$ and $r>0$ such that $2^{r} \underline{I}_{t o r}^{n}(X)=0$. Such an $r$ exists by unramifiedness of $\underline{I}_{t o r}^{n}$ and Lemma 29 
Step 1. If $m>r$, then $\log _{(m)}: F_{*}(X) \rightarrow \underline{I}_{t o r}^{*}(X)$ is a homomorphism of abelian groups. To see this, suppose that $x, y \in F_{n}(X)=F_{n} \underline{G W^{\times}}(X)$. Then $x^{P_{m}}=1+P_{m} x^{\prime}, y^{P_{m}}=1+P_{m} y^{\prime}$ for some (unique) $x^{\prime}, y^{\prime} \in \underline{I}_{\text {tor }}^{n}(X)$, and by definition $\log _{(m)}(x y)=z^{\prime}$, for the unique element $z^{\prime} \in \underline{I}_{\text {tor }}^{n}(X)$ such that $(x y)^{P_{m}}=1+P_{m} z^{\prime}$. We thus need to show that $\left(1+P_{m} x^{\prime}\right)\left(1+P_{m} y^{\prime}\right)=1+P_{m}\left(x^{\prime}+y^{\prime}\right)$. Note that for any $x^{\prime}, y^{\prime} \in \underline{I}_{\text {tor }}^{n}(X)$ we have $\left(1+P_{m} x^{\prime}\right)\left(1+P_{m} y^{\prime}\right)=1+P_{m}\left(x^{\prime}+y^{\prime}\right)+P_{m}^{2} x^{\prime} y^{\prime}$. Since $\left(\left\langle t_{i}\right\rangle-1\right)^{2}=-2\left(\left\langle t_{i}\right\rangle-1\right)$ we find that $P_{m}^{2}$ is divisible by $2^{m}$, and hence $P_{m}^{2} x^{\prime}=0$. This proves the claim.

Step 2. For $m>3 r$, the map $\log _{(m)}: F_{n}(X) \rightarrow \underline{I}_{t o r}^{n}(X)$ is a homomorphism of $\underline{G W}(X)$-modules. In other words we need to show that for $x \in F_{n} \underline{G W^{\times}}(X)$ and $y \in \underline{G W}(X)$ we have $y \log _{(m)}(x)=\log _{(m)}\left(x^{y}\right)$. By definition we have $x^{P_{m}}=1+P_{m} \log _{(m)}(x)$. Now

$$
1+P_{m} \log _{(m)}\left(x^{y}\right)=\left(x^{y}\right)^{P_{m}}=\left(x^{P_{m}}\right)^{y}=\left(1+P_{m} \log _{(m)}(x)\right)^{y}
$$

and so it is enough to show that $\left(1+P_{m} x^{\prime}\right)^{y}=1+y P_{m} x^{\prime}$ for every $x^{\prime} \in \underline{I}_{t o r}^{n}(X)$. By unramifiedness, we may assume that $X$ is the spectrum of a field $L$. Since $G W(L)$ is generated as an abelian group by 1 and the traces of quadratic extensions, and $\log _{(m)}$ is a homomorphism of abelian groups by step 1, it suffices to prove that for $L^{\prime} / L$ quadratic we have $\left(1+P_{m} x^{\prime}\right)^{\operatorname{tr}\left(L^{\prime}\right)}=1+\operatorname{tr}\left(L^{\prime}\right) P_{m} x^{\prime}$. By the base change and projection formulas, the left hand side equals $N_{L^{\prime}\left(t_{1}, \ldots, t_{m}\right) / L\left(t_{1}, \ldots, t_{m}\right)}\left(1+P_{m} x^{\prime}\right)$. Since $2^{r} x^{\prime}=0$ by assumption, the claim now follows from Corollary 39 and the projection formula.

Step 3. The sequence $\log _{(m)}(x)$ for $x \in F_{n}(X)$ is eventually constant. Let $m>3 r$. We compute

$$
x^{P_{m+1}}=\left(x^{P_{m}}\right)^{\left\langle t_{m+1}\right\rangle-1}=\left(1+P_{m} \log _{(m)}(x)\right)^{\left\langle t_{m+1}\right\rangle-1}=1+\left(\left\langle t_{m+1}\right\rangle-1\right) P_{m} \log _{(m)}(x),
$$

where in the last equality we have used step 2 . In other words we have found that $1+P_{m+1} \log _{(m+1)}(x)=$ $1+P_{m+1} \log _{(m)} x$. By the uniqueness part of Lemma 36, this means that $\log _{(m+1)}(x)=\log _{(m)} x$, which proves the claim.

We conclude that the limiting map $\log : F_{n} \rightarrow \underline{I}_{t o r}^{n}$ exists. It is easy to check that it is a homomorphism of (pre)sheaves of sets. By step 1 it is a homomorphism of sheaves of abelian groups.

Step 4. The limiting map $\log : F_{n}(X) \rightarrow \underline{I}_{t o r}^{n}(X)$ is compatible with transfers in the sense of equation (44). By unramifiedness we may assume that $X$ is the spectrum of a field. We need to prove that two elements in the lower right hand corner of equation (4) are equal. I claim that we may assume that $L^{\prime}=\prod_{i} L_{i}$, where each $L_{i}$ is an iterated quadratic extension over $L$. For this we use that (a) log is a map of presheaves, (b) transfers commute with base change, (c) $I^{n}(L) \rightarrow I^{n}\left(L^{\prime}\right)$ is injective if $\left[L^{\prime}: L\right]$ is odd (by Lemma 15), and then apply the argument from the proof of Proposition 17. Now that we have reduced to $L^{\prime}=\prod_{i} L_{i}$, the result follows from Corollary 39

Step 5. $\log$ is a morphism of homotopy modules. In other words log is compatible with the isomorphisms $\beta_{n}^{\dagger}: F_{n} \rightarrow\left(F_{n+1}\right)_{-1}$. To see this it is enough to show that $\log ([u] x)=[u] \log (x)$. But

$$
\log ([u] x)=\log \left(x^{\langle u\rangle-1}\right)=(\langle u\rangle-1) \log (x)=[u] \log (x) .
$$

Here the first and last equality are by definition, and the middle one is because log is a homomorphism of $G W$-modules, by step 2 .

Conclusion of proof for $v c d_{2}(k)<\infty$. We have already established (a), and (b) is step 5 . It is clear that for each $X, \log (X)$ is a bijection of sets, because each of the maps $\log _{(m)}(X)$ is a bijection. This proves (c). Property (d) was established in step 4.

Step 6. If $L$ is any field of characteristic $\neq 2$, and $x \in F_{n} G W^{\times}(L)$, then the sequence $\log _{(m)}(x)$ is eventually constant. By Corollary 51 there exist a subfield $l \subset L$ which is finitely generated over the prime field and $y \in F_{n} G W^{\times}(l)$, such that $x=\left.y\right|_{L}$. By step $3, \log _{(m)}(y)$ is eventually constant, and hence so is $\log _{(m)}(x)=\log _{(m)}\left(\left.y\right|_{L}\right)=\left.\log _{(m)}(y)\right|_{L}$. 
Conclusion for general $k$. We use the standard continuity argument. It follows from step 6 that $\log : F_{*} \rightarrow \underline{I}_{\text {tor }}^{*}$ is a well-defined map (of sheaves of sets, say). Then by Corollary 51 from Appendix B we have $\log (L)=\operatorname{colim}_{l} \log (l)$, where the colimit is over $l \subset L$ which are finitely generated over the prime field. Thus $v c d_{2}(l)<\infty$ for all such $l$, and hence $\log (l)$ is an isomorphism with all the desired properties (respects module structures and transfers), since we have already established the theorem in this case. It follows that the colimit $\log (L)$ has all the desired properties, too.

This concludes the proof.

Remark 41 . Let $k$ be a not necessarily perfect field and $p: \operatorname{Spec}(k) \rightarrow \operatorname{Spec}\left(k_{0}\right)$ be a morphism to a perfect field. Then $F_{*} \cong p^{*}\left(\left.F_{*}\right|_{k_{0}}\right)$ and $\underline{I}_{\text {tor }}^{*} \cong p^{*}\left(\left.\underline{I}_{\text {tor }}^{*}\right|_{k_{0}}\right)$, by the standard continuity arguments. It follows that the map $\log : F_{*} \rightarrow \underline{I}_{t o r}^{*}$ is a well-defined isomorphism of homotopy modules over $k$. If $G_{*}$ is a homotopy module over $k$ and $k$ is not perfect, then it is not clear (to the author) how to define transfers on $G_{*}$. If however $G_{*}$ is a homotopy module over $k_{0}$ then $p^{*} G_{*}$ is a homotopy module over $k$ which has canonical transfers. This applies to $F_{*}$ and $\underline{I}_{t o r}^{*}$, and we thus see that all the claims of Theorem 40 make sense and hold over $k$.

\section{Delooping $\underline{G W^{\times}}$}

In this section, we finally put everything together: we shall construct a homotopy module $T_{*}$ such that $T_{0}=\underline{G W^{\times}}$. We do this by taking the presentation

$$
I^{2}(k) \stackrel{a}{\rightarrow} G W(k) / 2 \oplus F_{2} G W^{\times}(k) \rightarrow G W^{\times}(k) \rightarrow 1
$$

from Proposition 25 and exhibiting a map $\tilde{a}: \underline{I}^{*+2} \rightarrow \underline{K}_{*}^{M W} / 2 \oplus F_{*+2}$ of homotopy modules such that $a=(\tilde{a})_{0}$.

Lemma 42. For any field $L$ of characteristic different from 2 we have the equality $2(\langle 2\rangle-1)=0 \in$ $G W(L)$.

Proof. Since $(x+y)^{2}+(x-y)^{2}=2\left(x^{2}+y^{2}\right)$ we have $\langle 1,1\rangle=\langle 2,2\rangle$. The result follows.

Lemma 43. The maps $\underline{I}^{n} \rightarrow F_{n} \underline{G W^{\times}}, x \mapsto(-1)^{x}$ assemble into a morphism of homotopy modules $\underline{I}^{*} \rightarrow F_{*}$. The composite

$$
\underline{I}^{*} \rightarrow F_{*} \stackrel{\log }{\longrightarrow} \underline{I}_{t o r}^{*}
$$

is multiplication by $\langle 2\rangle-1$.

Let us note that multiplication by $\langle 2\rangle-1$ is the zero map if $\sqrt{2} \in k$. Note also that in any case $\langle 2\rangle-1 \in G W(k)_{\text {tor }}$ by Lemma 42 .

Proof. We have $(-1)^{I^{n}} \subset F_{n} \underline{G W^{\times}}$by Proposition 23 part (iv), so the map exists as claimed. In order to see that we have a morphism of homotopy modules, we need to show that for $x \in \underline{I}^{n}(L)$ and $u \in L^{\times}$we have $(-1)^{[u] x}=[u]\left((-1)^{x}\right)$. Now $[u] x=(\langle u\rangle-1) x$ by the definition of $\underline{I}^{*}$ and so $(-1)^{[u] x}=\left((-1)^{x}\right)^{\langle u\rangle-1}$, which equals $[u]\left((-1)^{x}\right)$ by the definition of $F_{*}$ (see Corollary 34), as needed.

Recall that $\underline{I}^{*} \cong \underline{K}_{*}^{W}=\underline{K}_{*}^{M W} / h$, cf. [18]. Consider the composite map of homotopy modules $\underline{K}_{*}^{W} \cong \underline{I}^{*} \rightarrow F_{*} \stackrel{\log }{\longrightarrow} \underline{I}_{\text {tor }}^{*}$. Let $x \in I_{\text {tor }}^{0}(k)=W(k)_{\text {tor }} \subset W(k)$ be the image of $1 \in K_{0}^{W}(k)$. I claim that the composite $f: \underline{I}^{*} \rightarrow F_{*} \stackrel{\log }{\longrightarrow} \underline{I}_{t o r}^{*}$ is given by multiplication by $x$. Indeed if $y \in K_{*}^{M W}(L)$ then $f(y \cdot 1)=y \cdot f(1)=y \cdot x$, this being a map of homotopy modules. Since $\underline{K}_{*}^{M W} \rightarrow \underline{I}^{*}$ is surjective, this proves the claim. It remains to show that $x=\langle 2\rangle-1$. For this it is enough to prove that the composite $\underline{I}^{2} \rightarrow F_{2} \underline{G W^{\times}} \rightarrow \underline{I}_{t o r}^{2}$ is given by multiplication by $\langle 2\rangle-1$. Indeed then the two-fold contraction $\underline{W} \rightarrow \underline{W}_{t o r}$ will also be given by multiplication by $\langle 2\rangle-1$, so in particular $x=f(1)=\langle 2\rangle-1$.

Fix some field $L$. The ideal $I^{2}(L)$ is generated by elements of the form $(\langle a\rangle-1)(\langle b\rangle-1)$. It is thus enough to prove that

$$
(-1)^{(\langle a\rangle-1)(\langle b\rangle-1)\left(\left\langle t_{1}\right\rangle-1\right) \cdots\left(\left\langle t_{m}\right\rangle-1\right)}=1+(\langle 2\rangle-1)(\langle a\rangle-1)(\langle b\rangle-1)\left(\left\langle t_{1}\right\rangle-1\right) \cdots\left(\left\langle t_{m}\right\rangle-1\right)
$$


for all $m$. We prove this in two steps. First we deal with $m=0$. Let $A=k(\sqrt{a}), B=k(\sqrt{b})$. Then we compute

$$
\begin{array}{rlr}
(-1)^{(\langle a\rangle-1)(\langle b\rangle-1)} & =(-1)^{(\langle a\rangle+1)(\langle b\rangle+1)} & (-1)^{x}=(-1)^{-x} \\
& =(-1)^{\operatorname{tr}(A) \operatorname{tr}(B)} & \left(T_{A}\right),\left(T_{B}\right), 1=\langle 2\rangle^{2} \\
& =(\operatorname{tr}(A)-1)^{\operatorname{tr}(B)} & \\
& =(\langle 2\rangle(\langle a\rangle+1)-1)^{\operatorname{tr}(B)} & \\
& =(\langle a\rangle+1)^{\operatorname{tr}(B)}-\operatorname{tr}(A) \operatorname{tr}(B)+(-1)^{\operatorname{tr}(B)} & \left(T_{A}\right) \\
& =2+\langle a\rangle \operatorname{tr}(B)-\operatorname{tr}(A) \operatorname{tr}(B)+\operatorname{tr}(B)-1 & (* *),(* * *) \\
& =1+\operatorname{tr}(B)(1+\langle a\rangle)(1-\langle 2\rangle) & \left(T_{A}\right) \\
& =1+(\langle 2\rangle-1)(\langle a\rangle-1)(\langle b\rangle-1) \\
& =: 1+\xi .
\end{array}
$$

Here the right hand column justifies each manipulation, with the following abbreviations: $\left(T_{A}\right)$ means we use that $\operatorname{tr}(A)=\langle 2\rangle(\langle a\rangle+1),\left(T_{B}\right)$ means we use that $\operatorname{tr}(B)=\langle 2\rangle(\langle b\rangle+1),\left({ }^{*}\right)$ means we use that $\left.(x y)^{z}=x^{z} y^{z},{ }^{* *}\right)$ means we use that $\langle x\rangle^{\operatorname{tr}(B)}=1$ as follows from [29, Lemma 2.6(ii)], (***) means we apply Lemma 16, in the form that $(x+y)^{\operatorname{tr}(B)}=x^{\operatorname{tr}(B)}+y^{\operatorname{tr}(B)}+\operatorname{tr}(B) x y$, and $(* * * *)$ means we use that $x(\langle 2\rangle-1)=-x(\langle 2\rangle-1)$, which follows from Lemma 42. This proves the claim when $m=0$.

In order to prove the claim for $m>0$, it is enough to show that for any field extension $L^{\prime} / L$, and any $y \in G W\left(L^{\prime}\right)$ we have $(1+\xi)^{y}=1+y \xi$. Suppose that $(1+\xi)^{y_{i}}=1+y_{i} \xi$, for $y_{1}, y_{2} \in G W\left(L^{\prime}\right)$. Then $(1+\xi)^{y_{1}+y_{2}}=1+\left(y_{1}+y_{2}\right) \xi+y_{1} y_{2} \xi^{2}=1+\left(y_{1}+y_{2}\right) \xi$, provided that in addition $\xi^{2}=0$. Note that this condition is equivalent to $(1+\xi)^{2}=1+2 \xi$. Since $G W\left(L^{\prime}\right)$ is generated as an abelian group by 1 and the traces of quadratic extensions, it thus suffices to show that $(1+\xi)^{\operatorname{tr}(T)}=1+\operatorname{tr}(T) \xi$ for $T / L^{\prime}$ degree 2 , or equivalently that $\xi^{\operatorname{tr}(T)}=0$. We have $(\langle a\rangle-1)^{\operatorname{tr}(T)}=1-\operatorname{tr}(T)\langle a\rangle+\operatorname{tr}(T)-1=-\operatorname{tr}(T)(\langle a\rangle-1)$, by (***) with $T$ in place of $B$, and similarly for $b$ or 2 in place of $a$. Hence

$$
\xi^{\operatorname{tr}(T)}=(\langle 2\rangle-1)^{\operatorname{tr}(T)}(\langle a\rangle-1)^{\operatorname{tr}(T)}(\langle b\rangle-1)^{\operatorname{tr}(T)}=-\operatorname{tr}(T)^{3} \xi=-4 \operatorname{tr}(T) \xi=0,
$$

by Lemmas 42 and 38 (ii).

This concludes the proof.

Theorem 44. Let $k$ be a perfect field of characteristic different from 2. There exists a short exact sequence of homotopy modules

$$
0 \rightarrow \underline{K}_{*+2}^{W} /[-1] \underline{K}_{*+1}^{W} \stackrel{\eta^{2} \oplus(\langle 2\rangle-1)}{\longrightarrow} \underline{K}_{*}^{M W} / 2 \oplus \underline{K}_{t o r, *+2}^{W} \rightarrow T_{*} \rightarrow 0
$$

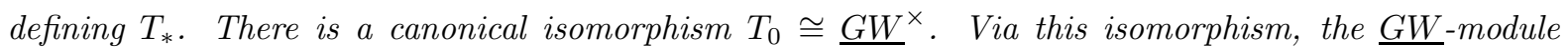
structure as well as the cohomological transfers on $T_{0}$ coincide with the module structure from Section 5 and Rost's multiplicative transfers, respectively.

Here by $[-1] \in K_{1}^{W}(k) \cong I(k)$ we denote the element $\langle-1\rangle-1$, and $\eta^{2}$ is just the natural inclusion $\underline{K}_{*+2}^{W}=\underline{I}^{*+2} \hookrightarrow \underline{K}_{*}^{M W}=\underline{I}^{*} \times_{\underline{k}_{*}^{M}} \underline{K}_{*}^{M}$. One may note that $\langle-1\rangle-1 \in I(k) \subset W(k)$ is the same as $-2 \in W(k)$.

Proof. Note that $\eta^{2}([-1])=\eta(-2)=-2 \eta=0 \in \underline{K}_{*}^{M W} / 2$, so the first map is well-defined, and that $(\langle 2\rangle-1)[-1]=(\langle 2\rangle-1)(\langle-1\rangle-1)=(\langle 2\rangle-1)(-2)=0 \in I(K) \subset W(K)$ by Lemma 42, so the second map is well-defined.

Being a sequence of homotopy modules, exactness in all degrees is equivalent to exactness in all sufficiently high degrees. Let us first show that $\underline{K}_{n+2}^{W} /[-1] \stackrel{\eta^{2} \oplus(\langle 2\rangle-1)}{\longrightarrow} \underline{K}_{n}^{M W} / 2 \oplus \underline{K}_{t o r, n+2}^{W}$ is injective, for $n \geq 0$. For this let $x \in K_{n+2}^{W}(L)$ and suppose that $\eta^{2}(x)=0$. Equivalently, we have $x \in I^{n+2}(L)$ with $\eta^{2}(x) \in 2 K_{n}^{M W}(L)$. Then in particular $x \in 2 I^{n}(L) \subset 2 W(L)$. By [2, Theorem 2.2] we conclude that $x \in 2 I^{n+1}(L)$. Note that this reference indeed applies, since $-2=\langle-1,-1\rangle \in W(L)$ is a Pfister form. Consequently $x \equiv 0 \in K_{n+2}^{W}(L) /[-1] K_{n+1}^{W}(L)$, i.e. $\eta^{2}$ alone is already injective.

I claim that we have, for every field $L / k$, a commutative diagram as follows

$$
\begin{array}{cccc}
K_{2}^{W}(L) /[-1] K_{1}^{W}(L) & \stackrel{\eta^{2} \oplus(\langle 2\rangle-1)}{\longrightarrow} & K_{0}^{M W}(L) / 2 \oplus K_{t o r, 2}^{W}(L) & \\
a \uparrow & b \oplus \log \uparrow & T_{0}(L) \\
I^{2}(L) & \stackrel{p \oplus q}{\longrightarrow} & G W(L) / 2 \oplus F_{2} G W^{\times}(L) \stackrel{r / s}{\longrightarrow} G W^{\times}(L) .
\end{array}
$$


Here we have used the notation of Proposition 25. The map $a$ is induced from the isomorphism $I^{2}(L) \cong$ $K_{2}^{W}(L)$, and the map $b$ is $G W(L) / 2 \cong K_{0}^{M W} / 2$. The claim is an immediate consequence of Lemma 43 .

Consider the map $r^{\prime} / s^{\prime}: \underline{K}_{0}^{M W} / 2 \oplus \underline{K}_{\text {tor }, 2}^{W} \cong \underline{G W} / 2 \oplus F_{2} \underline{G W}^{\times} \rightarrow \underline{G W}$. Here $F_{2} \underline{G W}^{\times} \cong \underline{I}_{\text {tor }}^{2} \cong$ $\underline{K}_{t o r, 2}^{W}$ is the composite of the logarithm isomorphism and Morel's isomorphism $\underline{I}^{*} \cong \underline{K}_{*}^{W}$ restricted to torsion, $r^{\prime}: \underline{G W} / 2 \rightarrow \underline{G W^{\times}}$is $x \mapsto(-1)^{x}$ and $s^{\prime}: F_{2} \underline{G W^{\times}} \rightarrow \underline{G W^{\times}}$is the inclusion. I claim that $r^{\prime} / s^{\prime}$ factors through $\underline{K}_{0}^{M W} / 2 \oplus \underline{K}_{t o r, 2}^{W} \rightarrow T_{0}$, and induces an isomorphism $T_{0} \cong \underline{G W^{\times}}$. Since we are dealing with unramified sheaves, both claims may be checked on sections over fields. There they follow from the commutativity of diagram (6), using that the map $a$ is surjective, $b \oplus \log$ is an isomorphism, and $r=r^{\prime}(L), s=s^{\prime}(L)$ under this isomorphism.

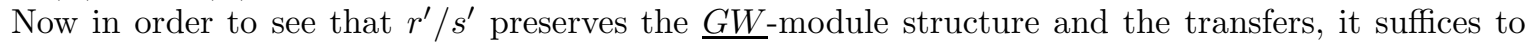
consider $r^{\prime}$ and $s^{\prime}$ separately. The fact that this works for $r^{\prime}$ follows from Proposition 22 parts (ii) and (iv). The map $s^{\prime}$ is the composite of the logarithm isomorphism, which preserves the module structure and transfers by Theorem 40, and the morphism of multiplication by a constant (namely $\langle 2\rangle-1)$ which also preserves the module structure and transfers. This concludes the proof.

Remark 45. If $\sqrt{2} \in k$ (i.e. $\langle 2\rangle=1 \in W(k))$ then we get a splitting $T_{*} \cong \underline{K}_{*}^{M W} /\left(2, \eta^{2}\right) \oplus \underline{K}_{\text {tor, } *+2}^{W}$, and in particular $G W^{\times}(k) \cong G W(k) /\left(2, I^{2}\right) \oplus I_{\text {tor }}^{2}(k)$, but not in general. This is essentially the same as Remark 24.

\section{A Recollections on homotopy modules}

Throughout, $k$ is a perfect base field. We recall some well-known facts about homotopy modules which seem hard to find explicitly in the literature. We make no claim to originality. Throughout $F_{*}$ will be an arbitrary homotopy module, with no necessary relation to the specific homotopy module constructed in Section 5 ,

The basics. Recall that for any (pre)sheaf $F$ (on $S m(k)$ ), its contraction is

$$
F_{-1}:=\underline{\operatorname{Hom}}\left(\mathbb{Z}\left[\mathbb{G}_{m}\right], F\right) \in \operatorname{Pre}(\operatorname{Sm}(k)) .
$$

Here $\mathbb{Z}\left[\mathbb{G}_{m}\right]=\mathbb{Z}\left[\left(\mathbb{A}^{1} \backslash 0,1\right)\right]$, where for a pointed scheme $(X, x)$ we put $\mathbb{Z}[(X, x)]=\mathbb{Z}[X] / \mathbb{Z}[\{x\}]$. Recall moreover that $F$ is called strictly homotopy invariant if for all $X \in S m(k)$ and $n \geq 0$, the canonical map $H_{N i s}^{n}(X, F) \rightarrow H_{N i s}^{n}\left(X \times \mathbb{A}^{1}, F\right)$ is an isomorphism.

A homotopy module [16, Section 5.2] consists of a collection of strictly homotopy invariant sheaves $F_{*} \in S h v_{N i s}(\operatorname{Sm}(k)), * \in \mathbb{Z}$ together with isomorphisms $F_{n} \rightarrow\left(F_{n+1}\right)_{-1}$. A morphism of homotopy modules $\alpha_{*}: F_{*} \rightarrow G_{*}$ consists of morphisms of sheaves $\alpha_{n}: F_{n} \rightarrow G_{n}$ for all $n$ such that the following diagram commutes for each $n$

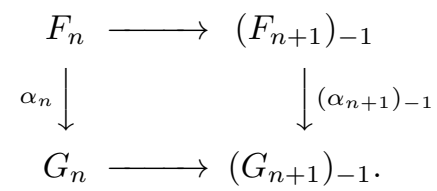

The category of homotopy modules is equivalent to the heart of the homotopy $t$-structure on $\mathbf{S H}(k)$ [16. Theorem 5.2.6]. This implies that they have a lot more structure than is immediately apparent. In this appendix we clarify some of this structure.

$\underline{K}_{*}^{M W}$-module structure. The object $\underline{\pi}_{0}(\mathbb{1})_{*} \cong \underline{K}_{*}^{M W}$ is the unit of a symmetric monoidal structure on the category of homotopy modules. For the definition of the sheaf of unramified Milnor-Witt $K$-theory $\underline{K}_{*}^{M W}$, see [20, Chapter 3]. Its sections over a field $L$ are generated by the classes $[u] \in K_{1}^{M W}(L)$ for $u \in L^{\times}$and $\eta \in K_{-1}^{M W}(L)$. One puts $\langle u\rangle=1+\eta[u]$; this induces an isomorphism $K_{0}^{M W}(L) \cong G W(L)$ [20, Lemma 3.10]. For each $n \geq 1$, the induced map $\mathbb{Z}\left[\mathbb{G}_{m}\right]^{\otimes n} \rightarrow \underline{K}_{n}^{M W}, u_{1} \otimes \cdots \otimes u_{n} \mapsto\left[u_{1}\right] \cdots\left[u_{n}\right]$ induces a surjection on sections over fields [20, Lemma 3.6].

Now suppose that $F_{*}$ is a homotopy module. The isomorphism $F_{n} \rightarrow\left(F_{n+1}\right)_{-1}$ corresponds by adjunction to a morphism $\mathbb{Z}\left[\mathbb{G}_{m}\right] \otimes F_{n} \rightarrow F_{n+1}$. Using the identification of the category of homotopy modules with the heart of $\mathbf{S H}(k)$, one may show that it factors through the surjection $\mathbb{Z}\left[\mathbb{G}_{m}\right] \rightarrow \underline{K}_{1}^{M W}$. By contraction, the pairing $\underline{K}_{1}^{M W} \otimes F_{n} \rightarrow F_{n+1}$ induces $\left(\underline{K}_{1}^{M W}\right)_{-2} \otimes F_{n} \rightarrow\left(F_{n+1}\right)_{-2}$ and hence a multiplication $\eta: F_{n} \rightarrow F_{n-1}$. Since $\underline{K}_{*}^{M W}$ is generated by $\underline{K}_{1}^{M W}$ and $\eta \in \underline{K}_{-1}^{M W}$, it follows that there is at most one extension to a pairing $\underline{K}_{n}^{M W} \otimes F_{m} \rightarrow F_{n+m}$ for all $m, n \in \mathbb{Z}$. It is a consequence of the 
identification of the category of homotopy modules with the heart of $\mathbf{S H}(k)$ that this extension always exists.

Cohomological transfers. A homotopy module automatically has cohomological transfers, i.e. for any finite étale morphism $f: X \rightarrow Y$ with $X, Y$ essentially smooth over $k$, there is a transfer $t r_{f}$ : $F_{*}(X) \rightarrow F_{*}(Y)$. See [20, Corollary 5.30] or [3, Section 4]. Cohomological transfers are functorial in morphisms of homotopy modules and satisfy the projection and base change formulas (loc. cit.). This means the following. If $a \in \underline{K}_{*}^{M W}(X)$ and $m \in F_{*}(Y)$, then $\operatorname{tr}_{f}\left(a f^{*} m\right)=\operatorname{tr}_{f}(a) \cdot m$, and similarly if $b \in \underline{K}_{*}^{M W}(Y)$ and $n \in F_{*}(X)$ then $\operatorname{tr}_{f}\left(f^{*}(b) n\right)=b t r_{f}(n)$; these are the projection formulas. Moreover if we have a cartesian square

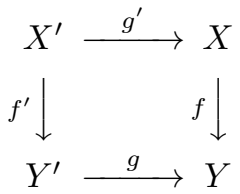

with $Y, Y^{\prime}$ smooth and $f$ étale, then $g^{*} t r_{f}(n)=\operatorname{tr}_{f^{\prime}} g^{\prime *}(n)$; this is the base change formula.

\section{More on contractions.}

Lemma 46. Let $t$ be a coordinate on $\mathbb{A}^{1}$ and $F_{*}$ a homotopy module. Write $i_{1}:$ Spec $(k) \rightarrow \mathbb{A}^{1} \backslash 0$ for the inclusion of the point 1 . Then for $X \in S m(k)$ we have $F_{*}\left(X \times\left(\mathbb{A}^{1} \backslash 0\right)\right) \cong F_{*}(X) \oplus F_{*-1}(X)$. Here the map $F_{*-1}(X) \rightarrow F_{*}\left(X \times\left(\mathbb{A}^{1} \backslash 0\right)\right)$ is multiplication by $[t] \in K_{1}^{M W}\left(k\left[t, t^{-1}\right]\right)$, the map $F_{*}(X) \rightarrow F_{*}\left(X \times\left(\mathbb{A}^{1} \backslash 0\right)\right)$ is pullback along the canonical projection, and the subgroup $F_{*-1}(X) \subset F_{*}\left(X \times\left(\mathbb{A}^{1} \backslash 0\right)\right)$ is precisely the kernel of $i_{1}^{*}$.

Proof. We have the inclusions $\operatorname{Spec}(k) \stackrel{i_{1}}{\rightarrow} \mathbb{A}^{1} \backslash 0 \rightarrow \mathbb{A}^{1}$. Since $F$ is homotopy invariant, it follows that $F_{*}\left(X \times\left(\mathbb{A}^{1} \backslash 0\right)\right)=F_{*}(X) \oplus M_{*}(X)$, where $M_{*}(X)$ is the kernel of $i_{1}^{*}$ and by definition of contraction, $M_{*}(X)=\left(F_{*}\right)_{-1}(X)$. We thus use the defining isomorphism of a homotopy module to identify $M_{*}(X) \cong$ $F_{*-1}(X)$. It remains to see that this isomorphism is given by multiplication by $[t]$.

For this, let $F$ be any homotopy invariant sheaf and $U \in S m(k)$ be arbitrary. If $X \in S m(k)$ then $\underline{\operatorname{Hom}}(\mathbb{Z}[X], F)(U)=F(X \times U)$ and the pairing $\operatorname{Hom}(U, X) \times \underline{\operatorname{Hom}}(\mathbb{Z}[X], F)(U) \rightarrow F(U)$ is given by $\alpha, x \mapsto \alpha^{* *}(x)$, where $\alpha^{\prime}=\left(\alpha, \operatorname{id}_{U}\right): U \rightarrow X \times U$ and $x \in F(U \times X)$. Since $F_{-1}(U)=$ $\underline{\operatorname{Hom}}\left(\mathbb{Z}\left[\mathbb{G}_{m}\right], F\right)(U) \subset F\left(\left(\mathbb{A}^{1} \backslash 0\right) \times U\right)$, the pairing $\mathbb{G}_{m}(U) \otimes F_{-1}(U) \rightarrow F(U)$ is given by $(u, s) \mapsto u^{\prime *} s$. Here $s \in F_{-1}(U) \subset F\left(U \times\left(\mathbb{A}^{1} \backslash 0\right)\right), u \in \operatorname{Hom}_{k}\left(U, \mathbb{A}^{1} \backslash 0\right)$ and $u^{\prime}=\left(u, \operatorname{id}_{U}\right) \in \operatorname{Hom}_{U}\left(U,\left(\mathbb{A}^{1} \backslash 0\right) \times U\right)$. Consequently "multiplication by $[t]$ "

$$
F\left(X \times\left(\mathbb{A}^{1} \backslash 0\right)\right) \supset F_{-1}(X) \rightarrow F_{-1}\left(X \times\left(\mathbb{A}^{1} \backslash 0\right)\right) \stackrel{[t]}{\longrightarrow} F\left(X \times\left(\mathbb{A}^{1} \backslash 0\right)\right)
$$

is given by $F\left(X \times\left(\mathbb{A}^{1} \backslash 0\right)\right) \supset F_{-1}(X) \ni s \mapsto t^{*} p^{*}(s)$, where $p: X \times\left(\mathbb{A}^{1} \backslash 0\right)^{2} \rightarrow X \times\left(\mathbb{A}^{1} \backslash 0\right)$ is projection to the first two factors and $t: X \times\left(\mathbb{A}^{1} \backslash 0\right) \rightarrow X \times\left(\mathbb{A}^{1} \backslash 0\right)^{2}$ is $(x, u) \mapsto(x, u, u)$. Thus $p t=\mathrm{id}$ and so multiplication by $[t]$ corresponds to the canonical inclusion, as was to be shown.

Boundary maps. Being strictly homotopy invariant, each of the sheaves $F_{n}$ is unramified [19, Lemma 6.4.4]. This means that for a dense open immersion $U \rightarrow X \in S m(k)$, the restriction $F_{*}(X) \rightarrow F_{*}(U)$ is injective and that moreover for $X$ connected we have

$$
F_{*}(X)=\bigcap_{x \in X^{(1)}} F_{*}\left(X_{x}\right)
$$

Here $X^{(1)}$ denotes the set of points of codimension one, $F_{*}\left(X_{x}\right)$ denotes the stalk at $x$, and the intersection takes place in $F_{*}(k(X))$.

If $L / k$ is a field extension, then by a standard colimit procedure there is a well-defined group of sections $F_{*}(L)$. More generally this is true if $L$ is a scheme which is a filtering inverse limit of a system of smooth schemes with affine transition morphisms. If $L$ is a finitely generated field extension of $k$ and $\mathcal{O} \subset L$ is a geometric dvr, then by definition there exist $X \in S m(k)$ and $x \in X^{(1)}$ such that $L \cong k(X)$ and $\operatorname{Spec}(\mathcal{O})$ is the localization of $X$ in $x$. Let $\kappa$ be the residue field of $\mathcal{O}$. Then for every choice of uniformizer $\pi$ of $\mathcal{O}$ there exists a canonical boundary map

$$
\partial^{\pi}: F_{*}(L) \rightarrow F_{*-1}(\kappa)
$$


with kernel $F_{*}(\mathcal{O})$ [20, discussion after Corollary 2.35].

In this situation, we write $s: \operatorname{Spec}(\kappa) \rightarrow \operatorname{Spec}(\mathcal{O})$ for the inclusion of the closed point.

Lemma 47. For $u \in \mathcal{O}^{\times}$and $m \in F_{*}(\mathcal{O})$ we have $\partial^{\pi}([u \pi] m)=\left\langle s^{*}(u)\right\rangle s^{*}(m)$.

Proof. First note that for $x \in K_{*}^{M W}(K)$ and $m \in F_{*}(\mathcal{O})$ we have $\partial^{\pi}(x m)=\partial^{\pi}(x) s^{*}(m)$. To see this, one goes back to the geometric construction of $\partial^{\pi}$ as in [20, Corollary 2.35]. That is we observe that after canonical identifications, $\partial^{\pi}$ corresponds to the boundary map $\partial: H^{0}\left(K, F_{*}\right) \rightarrow H_{v}^{1}\left(\mathcal{O}, M_{*}\right)$ in the long exact sequence for cohomology with support. Our claim then follows from the observation that for any sheaf of rings $K$ on a space $X$ and $K$-module $F$, the boundary map in cohomology with support satisfies our claim. To see this, just note that multiplication by $m \in F(X)$ induces a homomorphism of sheaves $K \rightarrow F \in \operatorname{Shv}(X)$ and consider the induced homomorphism of long exact sequences for cohomology with support.

We also have $\partial^{\pi}([u \pi])=\partial^{\pi}(\langle u\rangle[\pi]+[u])=\left\langle s^{*}(u)\right\rangle$, using [20, Lemma 3.5(1) and Proposition 3.17(3)] and the fact that the homomorphism $\partial^{\pi}$ has kernel $\underline{K}_{*}^{M W}(\mathcal{O})$.

This concludes the proof.

Lemma 48. If $G_{*} \hookrightarrow F_{*}$ is an inclusion of homotopy modules, then for any connected $X \in \operatorname{Sm}(k)$ we have $G_{*}(X)=F_{*}(X) \cap G_{*}(k(X))$.

Proof. Since $G_{*}$ is unramified we have $G_{*}(X)=\bigcap_{x \in X^{(1)}} G_{*}\left(X_{x}\right)$. It thus suffices to prove the lemma in case that $X=\operatorname{Spec}(\mathcal{O})$ with $\mathcal{O} \subset L$ a dvr with uniformizer $\pi$. Note that once a uniformizer $\pi$ has been fixed, the construction of the boundary map $\partial^{\pi}$ of a homotopy module is completely canonical, which implies that $\partial_{G}^{\pi}=\left.\partial_{F}^{\pi}\right|_{G}$ and hence $G_{*}(\mathcal{O})=\operatorname{ker}\left(\partial_{G}^{\pi}\right)=\operatorname{ker}\left(\partial_{F}^{\pi}\right) \cap G_{*}(L)$. This concludes the proof.

\section{B Recollections on continuity}

In this section we collect some continuity results which we use repeatedly, but could not find any references for. We again make no claims to originality.

By an essentially smooth $S$-scheme $X$ we mean a cofiltered diagram of $S$-schemes $X_{\alpha}, \alpha \in \Lambda$ with each $X_{\alpha} \rightarrow S$ smooth, and each transition map $X_{\alpha} \rightarrow X_{\beta}$ affine. By abuse of notation, we denote the limit $\lim _{\alpha} X_{\alpha}$ also by $X$. We call a morphism of schemes $X \rightarrow S$ essentially smooth if it can be obtained as the limit of a cofiltered diagram as above.

By an essentially finite type $S$-scheme $X$ we mean the same thing, except that $X_{\alpha} \rightarrow S$ is required to be of finite type instead of smooth.

Note that it follows from [9, Théorème 8.8.2(2) and Théorème 8.10.5(v)] [26, Tags 0C0C and 01OY] that essentially smooth (respectively essentially finite type) morphisms between Noetherian schemes are stable under composition.

Lemma 49. Suppose $X \rightarrow S$ is an essentially finite type morphism of Noetherian schemes. Then

$$
G W(X) \cong \operatorname{colim}_{\alpha} G W\left(X_{\alpha}\right)
$$

via the pullbacks $G W\left(X_{\alpha}\right) \rightarrow G W(X)$.

Proof. By [26, Tag 01ZR] the category of coherent sheaves on $X$ is the colimit of the categories of coherent sheaves on the $X_{\alpha}$. Any open subscheme of $X$ is the base change of an open subscheme of $X_{\alpha}$ for $\alpha$ sufficiently large [9, Théorème 8.8.2(2) and Théorème 8.10.5(iii)] and hence it follows easily that the category of vector bundles (locally free finite rank sheaves) on $X$ is also the colimit of the categories of vector bundles on the $X_{\alpha}$. The same result for the categories of bilinear bundles is now formal, and then $K(B i l(X))=\operatorname{colim}_{i} K\left(B i l\left(X_{\alpha}\right)\right)$.

By definition we have $G W(X)=K(\operatorname{Bil}(X)) / J(X)$, where $J(X)$ is the ideal consisting of elements $V-W$, where $V, W$ range over metabolic bilinear bundles with isomorphic Lagrangians $L$ [11, Section I.4]. Recall that $L \subset V$ being a Lagrangian means that $V=L \oplus L^{\perp}$.

It remains to show that $J(X)=\operatorname{colim}_{i} J\left(X_{\alpha}\right)$. This is immediate from the description of the category $\operatorname{Bil}(X)$ as the colimit of the categories $\operatorname{Bil}\left(X_{\alpha}\right)$.

Lemma 50. Let $s: X \rightarrow S$ be an essentially smooth morphism between Noetherian schemes of finite dimension, and $F \in \operatorname{Pre}(\operatorname{Sm}(S))$ a presheaf. For each $\alpha$ let $s_{\alpha}: X_{\alpha} \rightarrow S$ be the structure map. Then

$$
\left(s^{*} F\right)(X)=\operatorname{colim}_{\alpha} F\left(X_{\alpha}\right) .
$$

Moreover, $s^{*}$ preserves Nisnevich sheaves. 
Proof. This is a very special case of [10, Lemmas A.3 and A.4].

Corollary 51. If $K$ is a field, then $G W(K)=\operatorname{colim}_{k} G W(k)$, where $k$ runs through the subfields of $K$ which are finitely generated over the prime subfield. Such $k$ in particular have finite virtual 2-étale cohomological dimension.

More generally, let $p: X \rightarrow S$ be an essentially smooth morphism between Noetherian schemes of

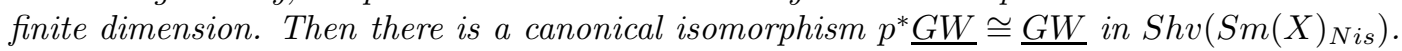

The same is true for $I^{n}$ or $W$ in place of $G W$.

Proof. Since $K=\bigcup_{k} k$ we have $\operatorname{Spec}(K)=\lim _{k} \operatorname{Spec}(k)$. Also the system is filtering with affine transition morphisms. Hence the first claim follows from Lemma 49] For the claim about cohomological dimension, see [25, Theorem 28 of Chapter 4].

For the more general statement, we note that the statement with the presheaf $G W$ in place of the sheaf $\underline{G W}$ follows from Lemmas 49 and 50 . So we need to show that $p^{*}$ commutes with taking the associated sheaf. This follows from [26, Tag 00WY].

Filtered colimits of abelian groups are exact, so the case of $I^{1}=\operatorname{ker}(G W \rightarrow \mathbb{Z})$ and $W=\operatorname{coker}(\mathbb{Z} \rightarrow$ $G W)$ follow from $G W$. For any filtering system $\left(R_{\alpha}, I_{\alpha}\right)$ of rings with a specified ideal we get $\operatorname{colim}_{\alpha} I_{\alpha}^{n} \cong$ $\left(\operatorname{colim}_{\alpha} I_{\alpha}\right)^{n}$, and hence we have established the claim about $I^{n}$. Finally the claims about $\underline{I}^{n}, \underline{W}$ are deduced from the results for $I^{n}, W$ as before.

Remark 52. Suitably formulated, the results in this section hold in much greater generality. We do not need this in the present article, so avoid the extra complications.

\section{References}

[1] Jón Kr Arason. Cohomologische Invarianten quadratischer Formen. Journal of Algebra, 36(3):448491, 1975.

[2] Jón Kr. Arason and Richard Elman. Powers of the Fundamental Ideal in the Witt Ring. Journal of Algebra, 239(1):150 - 160, 2001.

[3] Tom Bachmann. Motivic and Real Etale Stable Homotopy Theory. 2016. arXiv:1608.08855.

[4] Tom Bachmann and Marc Hoyois. Norms in Motivic Homotopy Theory. 2017. arXiv:1711.03061.

[5] Siegfried Bosch, Werner Lütkebohmert, and Michel Raynaud. Néron models, volume 21. Springer Science \& Business Media, 2012.

[6] Richard Elman and Christopher Lum. On the cohomological 2-dimension of fields. Communications in Algebra, 27(2):615-620, 1999.

[7] Daniel Ferrand. Un foncteur norme. Bulletin de la Société Mathématique de France, 126(1):1-49, 1998.

[8] Skip Garibaldi, Alexander Merkurjev, and Jean Pierre Serre. Cohomological invariants in Galois cohomology. Number 28. American Mathematical Soc., 2003.

[9] A Grothendieck and J Dieudonné. Éléments de géométrie algébrique IV. Publ. math. IHES, 1966.

[10] Marc Hoyois. From Algebraic Cobordism to Motivic Cohomology. Journal für die reine und angewandte Mathematik (Crelles Journal), 2013.

[11] Manfred Knebusch. Symmetric bilinear forms over algebraic varieties. In G. Orzech, editor, Conference on quadratic forms, volume 46 of Queen's papers in pure and applied mathematics, pages 103-283. Queens University, Kingston, Ontario, 1977.

[12] Simon Krsnik. Der Multiplikative Transfer auf dem Grothendieck-Witt Ring. Diplomarbeit, Universität Bielefeld, 2006.

[13] Tsit-Yuen Lam. The algebraic theory of quadratic forms. Lecture Notes Series in Mathematics. Benjamin/Addison-Wesley, 1973.

[14] Kristen Luise Mazur. On the Structure of Mackey Functors and Tambara Functors. PhD thesis, University of Virginia, 2013. 
[15] John Willard Milnor and Dale Husemoller. Symmetric bilinear forms, volume 60. Springer, 1973.

[16] Fabien Morel. An introduction to $\mathbb{A}^{1}$-homotopy theory. ICTP Trieste Lecture Note Ser. 15, pages 357-441, 2003.

[17] Fabien Morel. On the motivic $\pi_{0}$ of the sphere spectrum. In Axiomatic, enriched and motivic homotopy theory, pages 219-260. Springer, 2004.

[18] Fabien Morel. Sur les puissances de l'idéal fondamental de l'anneau de Witt. Commentarii Mathematici Helvetici, 79(4):689-703, 2004.

[19] Fabien Morel. The stable $\mathbb{A}^{1}$-connectivity theorems. K-theory, 35(1):1-68, 2005.

[20] Fabien Morel. $\mathbb{A}^{1}$-Algebraic Topology over a Field. Lecture Notes in Mathematics. Springer Berlin Heidelberg, 2012.

[21] Manuel Ojanguren and Ivan Panin. A purity theorem for the Witt group. Annales Scientifiques de l'École Normale Supérieure, 32(1):71 - 86, 1999.

[22] Markus Rost. The Multiplicative Transfer for the Grothendieck-Witt Ring. preprint, 2003.

[23] Joseph Rotman. An introduction to the theory of groups, volume 148. Springer Science \& Business Media, 2012.

[24] Winfried Scharlau. Quadratic and Hermitian forms, volume 270. Springer Science \& Business Media, 1985.

[25] S.S. Shatz. Profinite Groups, Arithmetic, and Geometry. Annals of mathematics studies. Princeton University Press, 1972.

[26] The Stacks Project Authors. Stacks Project. http://stacks.math.columbia.edu, 2017.

[27] D. Tambara. On multiplicative transfer. Communications in Algebra, 21(4):1393-1420, 1993.

[28] Matthias Wendt. Units in Grothendieck-Witt rings and $\mathbb{A}^{1}$-spherical fibrations. arXiv preprint arXiv:1304.5922, 2013.

[29] Tobias Wittkop. Die Multiplikative Quadratische Norm für den Grothendieck-Witt-Ring. Diplomarbeit, Universität Bielefeld, 2006. 